
\title{
$\begin{array}{ll}\text { Research Square } & \begin{array}{l}\text { Preprints are preliminary reports that have not undergone peer review. } \\ \text { They should not be considered conclusive, used to inform clinical practice, } \\ \text { or referenced by the media as validated information. }\end{array}\end{array}$
}

\section{Novel Diagnostic and Therapeutic Techniques Reveal Changed Metabolic Profiles in Recurrent Focal Segmental Glomerulosclerosis}

Janina Müller-Deile ( $\sim$ janina.mueller-deile@uk-erlangen.de)

Department of Nephrology and Hypertension, Friedrich-Alexander-University (FAU) Erlangen-Nuremberg

\section{George Sarau}

Fraunhofer Institute for Ceramic Technologies and Systems

\section{Christoph Daniel}

Department of Nephropathology, Friedrich-Alexander-University (FAU) Erlangen-Nuremberg

\section{Christian Jaremenko}

Institute of Optics, Information and Photonics, Friedrich-Alexander-University (FAU) Erlangen-Nuremberg, Germany

\section{Ahmed M Kotb}

Department of Nephrology and Hypertension, Friedrich-Alexander-University (FAU) Erlangen-Nuremberg

\section{Stefan Kalkhof}

Institute for Bioanalysis, University of Applied Sciences Coburg

\section{Ulrike E Rolle-Kampczyk}

Protein biomarker unit, Department of Therapy validation, Fraunhofer Institute for Cell Therapy and Immunology

\section{Silke Christiansen}

Fraunhofer Institute for Ceramic Technologies and Systems

\section{Mario Schiffer}

Department of Nephrology and Hypertension, Friedrich-Alexander-University (FAU) Erlangen-Nuremberg

\section{Research Article}

Keywords: Recurrent FSGS, circulating permeability factors, Raman spectroscopy, mass spectrometry, zebrafish, CytoSorb apheresis, metabolomic change

Posted Date: November 25th, 2020

DOI: https://doi.org/10.21203/rs.3.rs-109697/v1

License: (c) (i) This work is licensed under a Creative Commons Attribution 4.0 International License.

Read Full License 
Version of Record: A version of this preprint was published at Scientific Reports on February 25th, 2021. See the published version at https://doi.org/10.1038/s41598-021-83883-w. 


\section{Abstract}

Idiopathic forms of Focal Segmental Glomerulosclerosis (FSGS) are caused by circulating permeability factors, which can lead early recurrence of FSGS and kidney failure after kidney transplantation. In the past three decades, many research endeavors were undertaken to identify these unknown factors. Even though some potential candidates have been recently discussed in the literature, "the" actual factor remains elusive. Therefore, there is an increased demand in FSGS research for the use of novel technologies that allow us to study FSGS from a yet unexplored angle. Here, we report the successful treatment of recurrent FSGS in a patient after living related kidney transplantation by removal of circulating factors with CytoSorb apheresis. Interestingly, the classical published circulating factors were all in normal range in this patient but early disease recurrence in the transplant kidney and immediate response to CytoSorb apheresis were still suggestive for pathogenic circulating factors. To proof the functional effects of the patient's serum on podocytes and the glomerular filtration barrier we used a podocyte cell culture model and a proteinuria model in zebrafish to detect pathogenic effects on the podocytes actin cytoskeleton inducing a functional phenotype. We then performed Raman spectroscopy in the $<50 \mathrm{kD}$ serum fraction, on cultured podocytes treated with the FSGS serum and in kidney biopsies of the same patient at the time of transplantation and at the time of disease recurrence. The analysis revealed changes in podocyte metabolom induced by the FSGS serum as well as in focal glomerular and parietal epithelial cell regions in the FSGS biopsy. Several altered Raman spectra were identified in the fractionated serum and metabolome analysis by mass spectrometry detected lipid profiles in the FSGS serum, which were supported by disturbances in the Raman spectra. Our novel innovative analysis reveals changed lipid metabolome profiles associated with idiopathic FSGS that might reflect a new subtype of the disease.

\section{Introduction}

Primary focal segmental glomerulosclerosis (FSGS) is a rare disease with an estimated incidence of about 7 per 1 million ${ }^{1}$. However, it is still one of the most prevalent nephropathies causing end-stage renal disease ${ }^{2,3}$. FSGS is a morphologic pattern of glomerular injury defined by the presence of sclerosis in parts (segmental) of some (focal) glomeruli and global podocyte foot process effacement in all glomeruli. Subclasses of FSGS include primary (idiopathic), genetic and secondary forms. Despite sharing certain clinical and histologic features, these subclasses differ in management and prognosis ${ }^{4}$. Distinguishing between different forms of FSGS is important to avoid unnecessary immunosuppressive treatments in case of secondary forms and most genetic forms ${ }^{5}$. Secondary FSGS is the consequence of other underlying chronic diseases such as diabetes, obesity, HIV, hypertension and other glomerular diseases. Genetic FSGS results from defects in podocyte genes and is often steroid resistant. Mutations in more than 30 recessive or dominant genes were identified for monogenic forms of steroid resistant nephrotic syndrome. Recessive mutations in nephrin (NPHS1), podocin (NPHS2), CD2 associated protein (CD2AP), laminin $\beta 2$ (LAMB2), integrin, phospholipase $C$ epsilon 1 (PLCE1), protein tyrosine phosphatase receptor type 0 (PTPRO) and alpha 3 (ITGA3) are only some examples. Dominant mutations include actin 
alpha 4 (ACTN4), inverted formin 2 (INF2), anillin (ANLN), transient receptor potential cation channel (TRPC6) and Wilms tumor 1 (WT1) ${ }^{6}$.

In contrast, idiopathic FSGS-forms are most likely caused by circulating permeability factors. Soluble urokinase plasminogen activator receptor (SUPAR), cardiotrophin-like cytokine factor-1 (CLCF1) and SCD25 have been postulated as such permeability factors in FSGS ${ }^{7-10}$. However, it remains to be determined whether these factors are truly pathogenic, specific, sensitive and reproducible in all patients and just like the genetic forms it is likely that this disease category represents only a subgroup of diseases and that not only one but several different or a combination of circulatory factors exist.

The incidence of FSGS is increasing across the entire age spectrum worldwide ${ }^{11}$. Rapid onset of heavy proteinuria in a nephrotic range, hypoalbuminemia and edema are typical symptoms and sings of primary FSGS. FSGS non-responsive to steroid treatment has a high risk to progress to end stage kidney disease and second line treatment is often not effective or disrupted due to side effects. Moreover, patients who progress to end stage kidney disease and receive a kidney transplant have a 20 to $30 \%$ risk of FSGS recurrence in their kidney graft ${ }^{11,12}$.

There is an unmet clinical need for early and specific biomarkers to detect FSGS and to predict its prognosis and response to therapy. So far, diagnostic and therapeutic decisions are based on unspecific markers as proteinuria, serum creatinine as well as renal histology. However, even by histology the actual disease of the patients might be misinterpreted, as FSGS is a focal disease vulnerable to sampling errors in the biopsy ${ }^{13}$.

Here we report CytoSorb apheresis as a novel therapeutic approach to bring recurrent FSGS in remission in a patient that was nonresponsive to immunosuppressive therapy and plasmapheresis. We report an innovative zebrafish model to detect the presence of circulating permeability factors causing FSGS that might be used as non-invasive diagnostic test in the future. In order to characterize idiopathic FSGS on cell, serum and tissue level, we performed Raman spectroscopy in podocytes treated with the patient's serum and control serum, in $<50 \mathrm{kD}$ a serum fractions for FSGS patient and a control patient and on renal biopsies of a patient at the time of kidney transplantation and at the time of FSGS recurrence. Assignment of Raman signals in the FSGS biopsy to published metabolites corresponded to mass spectrometry findings. Serum metabolome analysis was able to detect metabolic changes in the patient's serum during the active phase of the disease. Previously described circulating factor were detectable in the normal range. Therefore, we hypothesize that these metabolomic signatures indirectly reflects the effects of the unknown circulating factors in the kidney or that these metabolome changes might even represent the pathogenic components themselves.

\section{Results}

\section{CytoSorb apheresis to treat for treatment resistant and recurrent FSGS}


Treatment options for FSGS caused by circulating factors are limited and often unsuccessful. We report a 24-year-old woman with nephrotic syndrome and biopsy proven FSGS in her native kidneys (Fig. 1A a, b). Clinical history for secondary FSGS was negative and genetic testing did not reveal reported mutations in podocyte genes. Different treatment regimens including steroids, cyclosporine A, mycophenolate mofetil, rituximab and even plasmapheresis were unsuccessful and within 12 months after initial diagnosis, the patient developed ESRD and was started on hemodialysis. Two years later, the patient received a livingrelated donor kidney transplantation from her mother. Primary renal transplant function was very good. Serum creatinine decreased to $1.14 \mathrm{mg} / \mathrm{dl} 10$ days after transplantation and no significant proteinuria was detectable. However, one month after transplantation, the patient developed proteinuria with a urinecreatinine-protein-ratio (UPC-ratio) of $3 \mathrm{~g} / \mathrm{mg}$ creatinine. Transplant kidney biopsy showed podocytopathy with podocyte effacement, which confirmed recurrent disease (Fig. 1A c, d). The patient received intravenous corticoid treatment and two doses of rituximab $(1 \mathrm{~g})$ within 2 weeks. Despite of this therapy proteinuria did not respond. Given the early recurrence of significant proteinuria shortly after transplantation, we hypothesized the presence of a circulating permeability factor causing the disease. Due to the severity and rapid progression of the primary disease, the early recurrence after transplantation and the past medical history where the disease in the native kidneys was refractory to established treatment regimens, we decided to treat the patient with CytoSorb apheresis as a compassionate use approach. Initially, CytoSorb apheresis was performed daily. Proteinuria immediately decreased to 327 $\mathrm{mg} / \mathrm{g}$ creatinine and CytoSorb apheresis treatment schedule was reduced to once a week (Fig. 1B). However, proteinuria rapidly relapsed to $4235 \mathrm{mg} / \mathrm{g}$ creatinine so that CytoSorb treatment frequency was increased again until the patient developed clinical remission. However, a week later proteinuria again relapsed (3686 mg/g creatinine) (Fig. 1B). A third time CytoSorb apheresis was performed daily and brought the patient back into remission. After additional therapy with rituximab, CytoSorb treatment was tapered to once a week. Since then, the patient was in remission with proteinuria around $0.5 \mathrm{mg} / \mathrm{g}$ creatinine and serum creatinine around $1.5 \mathrm{mg} / \mathrm{dl}$ under CytoSorb treatment every second week. We follow this patient now for more than two years with an excellent transplant function and have so far not been able to wean the patient completely from CytoSorb apheresis. All attempts to increase the time between the treatment cycles have failed. Of note, if the patient is missing s session (e.g. due to a holiday) proteinuria immediately increases and rituximab treatments had to continue in six months intervals (data not shown). We measured proposed circulating permeability factors in the patient's serum before and after first, second and third CytoSorb apheresis. SUPAR $(<4000 \mathrm{pg} / \mathrm{ml})$ and $\operatorname{sCD} 25(<500$ $\mathrm{U} / \mathrm{ml}$ ) were in normal rage before the first apheresis and decreased not significantly in response to CytoSorb treatments (Fig. 1C a, b). Serum concentration of CLCF1 was $1.67 \mathrm{ng} / \mathrm{ml}$ at the time of FSGS recurrence and decreased to $0.82 \mathrm{ng} / \mathrm{ml}$ after the 3 . CytoSorb treatment. Using the same ELISA in serum samples form healthy controls, concentrations of CLCF1 were between $0 \mathrm{ng} / \mathrm{ml}$ ( $66 \%$ of controls) and 1.2 $\mathrm{ng} / \mathrm{ml}$. Cathepsin L (undetectable) and IL6 $(<2 \mathrm{pg} / \mathrm{ml}$ ) were also not elevated in the patient's serum (data not shown).

\section{Ex vivo tests to detect morphological changes in podocytes caused by FSGS serum}


Even though published circulating factors were in normal range or not detectable in our patient, the early disease recurrence after kidney transplantation and the immediate response to CytoSorb treatment were still suggestive or a disease-causing factor in the patient's circulation. To screen for functional impacts of the patient's serum on podocytes we used a cell-based ex vivo screening model. Differentiated human podocytes were cultured in the presence of serum from our patient with FSGS recurrence (FSGS serum) and compared to podocytes cultured in the presence of control serum (CTRL serum) for three and six hours. Cytoskeletal rearrangements with a general loss of central stress fibers were detected after podocytes were exposed to serum derived from our patient with recurrent FSGS. (Fig. 2A). In contrast, serum from a nephrotic patient with membranous glomerulonephritis did not induce cytoskeletal changes (data not shown). Thus, the in vitro assay indicates the presence of unknown pathogenic circulating factors. To test if these factors would also induce increased permeability of the glomerular filtration barrier, we used our zebrafish model for proteinuria screening. The zebrafish is an ideal model system for glomerular diseases as the zebrafish larvae develops a pronephros which is morphological almost indistinguishable from the human glomerulus and genes are highly conserved between zebrafish and human. In the past, we successfully used this zebrafish model to screen for proteinuria after genetic knockdown using morpholinos ${ }^{14-21}$. We usually use a transgenic zebrafish line that expresses a fluorescent Vitamin D binding plasma protein Tg(l-fabp:VDBP:eGFP fish) to indirectly measure proteinuria 22. If plasma proteins are retained in the vascular system, the fluorescent signal from the Vitamin $D$ binding protein can easily be detected in the retinal vessels of the zebrafish (Fig. 2Ba, C). In case of proteinuria, the fluorescent signal decreases ${ }^{14-18,23}$. Given the idea of an unknown circulating permeability factor, we refined our zebrafish model by injecting patient serum that was mixed 1:1 with red fluorescent dextran to control proper injection procedure in the zebrafish circulation at 48 hours post fertilization (hpf) (Fig. 2Ba,b). Compared to zebrafish that were injected with serum from a healthy control, zebrafish injected with serum from the patient with recurrent FSGS developed lost high molecular weight plasma proteins at $120 \mathrm{hpf}$ (Fig. 2Bc). Taken together these tests proved that our patients FSGS serum contains a so far unknown permeability factor that acts on the podocyte cytoskeleton and changes the permeability of the glomerular filtration barrier.

\section{Raman spectroscopy reveals molecular fingerprint of FSGS serum and changes in podocyte metabolome induced by FSGS serum}

In order to further characterize this unknown factor, we used a novel technique, which has so far not been used in the context of FSGS, Raman spectroscopy. This spectroscopy method is a technique for optical characterization of the compositional properties of materials. It detects the inelastic scattering of light from molecules, which results in a change in wavelength that corresponds to specific molecular vibrational modes. As CytoSorb therapy eliminates molecules with a molecular weight below $50 \mathrm{kDa}$, we analyzed the $<50 \mathrm{kDa}$ serum fractions of the FSGS patient before the first CytoSorb apheresis at the time of FSGS recurrence and at the time of remission as well as in a control person by Raman spectroscopy. Raman shifts corresponding to phosphatidylcholine $\left(\sim 876 \mathrm{~cm}^{-1}, 1065 \mathrm{~cm}^{-1}\right)$, L-carnitine $\left(1461 \mathrm{~cm}^{-1}\right)$, C=C-lipids and amid $1\left(16601065 \mathrm{~cm}^{-1}\right)$ were elevated at the time of FSGS recurrence. In contrast, 
Raman signals of the $<50 \mathrm{kDa}$ serum fraction at time of FSGS remission resembled the Raman signal of $<50 \mathrm{kDa}$ serum fraction of a control person (table 1 and Fig. 3A). Thus, Raman spectroscopy revealed a changed serum lipid metabolome in FSGS. Raw data of Raman intensity and Raman shift can be found in supplementary table 1.

To explore the molecular basis of the patient's serum-induced morphological changes on podocytes further we performed Raman spectroscopy of cultured podocytes treated with FSGS serum and control serum. Interestingly, the Raman signal intensity of cells treated with control serum for $6 \mathrm{~h}$ was less intense compared to podocytes treated with FSGS serum (Fig. 3Ba-d).

Among others, increased Raman signal shifts assigned to membrane bound phosphatidylcholine ( 720/ $\left.1002 \mathrm{~cm}^{-1}\right)$, to choline $\left(\sim 760 \mathrm{~cm}^{-1}\right)$, phenylalanine, $\left(1003 / 1030 \mathrm{~cm}^{-1}\right)$, fatty acids $(\mathrm{a}(\mathrm{CH} 2 / \mathrm{CH} 3)$ and $\beta(\mathrm{CH} 2 / \mathrm{CH} 3)$ at $\left.\sim 1433 / 1457 \mathrm{~cm}^{-1}\right)$ and a sphingolipid cluster $\left(\sim 1650 \mathrm{~cm}^{-1}\right)$ were detectable in podocytes treated with FSGS serum (table 1). Raw data of Raman signal intensity and Raman signal shift can be found in supplementary table 2.

Taken together, changes in Raman signals in FSGS treated cultured human podocytes could mostly be attributed to cellular lipoproteins.

\section{Raman spectroscopy gives a molecular fingerprint of recurrent FSGS on tissue level}

To explore if we can also get a molecular fingerprint of recurrent FSGS on tissue level, we performed Raman micro-spectroscopy mapping on different glomerular regions of a kidney graft biopsy at time of transplantation (0 biopsy) (Fig. 4Aa, b) and at the time of FSGS relapse (FSGS recurrence) (Fig. 4Ac, d). Interestingly, increased Raman signal was detected in the region of parietal epithelial cells in the Bowman's capsule in the biopsy with FSGS recurrence indicating activation of these cells (arrowheads in Fig. 4Ad). We again identified dominant peaks of Raman signalsin the FSGS biopsy corresponding to biochemical such as membrane bound phosphatidylcholine at $766 \mathrm{~cm}^{-1}$, phenylalanine at $\sim 1003 \mathrm{~cm}^{-1}$, collagen at $\sim 1259 \mathrm{~cm}^{-1}$, and amide I of sphingomyelin at $\sim 1660 \mathrm{~cm}^{-1}$. By contrast, Raman peaks at $1065 \mathrm{~cm}^{-1}$ (cholesterol), $1128 \mathrm{~cm}^{-1}(\mathrm{C}-\mathrm{N}), 1303 \mathrm{~cm}^{-1}$ (amide III, cytosine, adenine) and $1448 \mathrm{~cm}^{-1}(\mathrm{CH} 2$ protein) were reduced in FSGS biopsy compared to the biopsy at transplantation (Fig. 3Be, table 1). This indicates an altered amino acid and lipid composition in the kidney with FSGS recurrence. To analyze anomalies in Raman spectra between 0-biopsy and FSGS recurrence we generated a Raman spectroscopy map from two measurements belonging to each biopsy and used a machine learning approach (One-Class SVM) (Fig. 4B). Raman spectra were visualized, whereby the intensity and color range cover the degree of the Raman anomaly. Areas of parietal epithelial cells in the Bowman capsule (white arrows in Fig. 4B) as well as focal glomerular lesions (white arrows in Fig. 4B) could be detected as an anomaly in the FSGS samples. Dividing the whole Raman signal into only parts of the spectrogram revealed that there were significant differences in focal areas of glomeruli in the FSGS recurrence biopsy (dotted red line in in Fig. 4B a'-d') compared to the 0-biopsy (blue line in in Fig. 4B a'-d'). This was most prominent in the wavelength range of $775-1160 \mathrm{~cm}^{-1}, 1138-1523 \mathrm{~cm}^{-1}$ as well as $1500-1800 \mathrm{~cm}^{-1}$. 
Anomaly spectra from the parietal cell region of the bowman capsule were most prominent in wavelength range of $775-1160 \mathrm{~cm}^{-1}$ (red line in Fig. 4B a'-d'). Raw data of Raman intensity and Raman shift can be found in supplementary table 3.

\section{Serum metabolome analysis of FSGS serum reveals potential disease-causing factors}

To characterize the patient's serum further we performed mass spectrometry analysis. 163 metabolites and metabolite ratios were analyzed by a targeted mass spectrometry approach in the FSGS patient's serum at different time points as well as in serum from a stable transplanted control patient.

Ornithine/arginine ratio, ornithine, different lysophosphatidylcholines, tyrosine phosphatidylcholines as well as proline were increased in the FSGS serum at the time of relapse in the kidney transplant compared to serum of a stable transplanted patient (log2 fold change > 0.3) (table 2). In contrast, different acylcarnitines $(\mathrm{CO}, \mathrm{C} 2, \mathrm{C} 3-\mathrm{DC}(\mathrm{C} 4-\mathrm{OH}))$ and tyrosine/ phenylalanine ratio were decreased in serum of the FSGS patient compared to serum of the control patient (log2 fold change < -0.5 (table 2). Comparing the metabolite abundances at the time of FSGS remission to that at the time of recurrence revealed a decrease of initially elevated lysophosphatidylcholines and an increase in initially reduced acylcarnitines (table 3). This change was significant for L-carnitine C0 (log2 fold change $=+1.33$; $-\log 10$ ( $p$-value) $=$ 0.009) and phosphatidylcholine aaC34:4 (log2 fold change of -0.75 , - $\log 10$ ( $p$-value) = 0.029) (Fig. 5). Comparing different metabolite classes after the first CytoSorb apheresis to that before the first CytoSorb apheresis showed a decrease in amino acids initially increases in FSGS serum and an increase in acylcarnitine initially reduced (table 4). CytoSorb apheresis in a stable phase of FSGS further decreased lysophosphatidylcholines and phosphatidylcholines that were still elevated compared to the control patient (table 5). Raw data of mass spectrometry analysis normalized by the control serum can be found in supplementary table 4.

In summary, mass spectrometry analysis revealed changes in serum lipoprotein profile in FSGS that could be influenced by CytoSorb therapy. Furthermore, mass spectrometry results correlated to Raman signals found in FSGS serum treated podocytes, FSGS serum fractions and FSGS kidney biopsy.

\section{Discussion}

Idiopathic FSGS is a disease group that is believed to be caused by circulating permeability factors. Despite long lasting research efforts over many decades and several identified potential factors a unifying concept has not been established for circulating factors. Here, we report a patient with recurrent idiopathic FSGS negative for all published circulating permeability factors. Since we previously published successful removal of sUPAR treatment with CytoSorb apheresis in a patient with FSGS and since our patient had progressed to end-stage renal disease of her native kidneys despite all other treatment attempts we decided to use CytoSorb as compassionate use ${ }^{24}$. CytoSorb preferentially absorbs hydrophobic substances and remove molecules with a weight range between 5-60 kDa. Already one treatment with CytoSorb apheresis led to rapid decrease in proteinuria with no side effects in this patient. However, at the beginning of the treatment proteinuria relapsed shortly after apheresis frequency was 
reduced. This might indicate the rapid rebound of the circulating components after CytoSorb treatment. Only after daily treatment sessions over several weeks and additional rituximab treatment CytoSorb apheresis sessions could be reduced to once a week. Even though the patient achieved remission with our treatment, the actual circulating component in the patient's blood remains unknown.

There is plenty of evidence for a causative circulating factor in idiopathic FSGS: Serum from patients with FSGS increases glomerular albumin permeability in vitro and induces proteinuria in rats ${ }^{25,26}$. Proteinuria can recur days to weeks after kidney transplantation and plasmapheresis is able to induce proteinuria remission ${ }^{27,28}$. Implantation of a kidney allograft with FSGS in another patient was successful without causing proteinuria ${ }^{29}$. One of the most prominent, but also most debated circulating factor suggested to cause FSGS is SUPAR ${ }^{30,31}$. sUPAR is released during inflammation or immune activation, and therefore the sUPAR levels reflect immune activation ${ }^{32}$. Normal sUPAR level range from $2000-3000 \mathrm{pg} / \mathrm{mL}$ in healthy individuals, about $3000-4000 \mathrm{pg} / \mathrm{mL}$ in unselected patients in emergency departments, and about $9000-10000 \mathrm{pg} / \mathrm{mL}$ in critically ill patients. sUPAR levels are higher in females compared to males and smoking is associated with an increase in sUPAR compared to non-smokers ${ }^{33}$. We could show in a previously published case report, that CytoSorb treatments can decrease sUPAR levels ${ }^{24}$. However, our recent patient showed sUPAR levels in a normal range. CLCF1 is another putative circulating permeability factor described in FSGS ${ }^{34}$. Recombinant human CLCF1 increased albumin permeability of isolated rat glomeruli ${ }^{35}$. Incubation of cultured murine podocytes with CLCF1 caused marked changes in the configuration of the actin cytoskeleton ${ }^{36}$. Serum CLCF1 concentration was 1.67 $\mathrm{ng} / \mathrm{ml}$ at the time of FSGS recurrence in our patient. Even though undetectable in most of our healthy controls, serum CLCF1 reached concentrations up to $1.2 \mathrm{ng} / \mathrm{ml}$ in one control. Therefore, we do not believe that CLCF1 is specific for recurrent FSGS and most likely was not the disease-causing factor in our patient. Kemper et al. observed increased levels of the T-cell activation marker SCD25 during relapses of steroid dependent nephrotic syndrome ${ }^{37}$. SCD25 level were in normal range CD25 level before the first apheresis session in our patient. Cathepsins are other suggested candidates for the circulating permeability factor. Cathepsins are proteases involved in intracellular protein degradation and activation of enzyme precursors. Immunohistochemically staining of human kidney biopsy specimens indicated that the expression of cathepsin D was significantly increased in Minimal change disease compared to that in FSGS maybe because of a high level of autophagic activity ${ }^{38,39}$. IL 6 was demonstrated to contribute to renal diseases like FSGS ${ }^{40}$. Cathepsin L and IL 6 were undetectable or in normal range in in our patient.

Since our patient had normal range of published circulating permeability factors, we used our cell culture model and our zebrafish proteinuria assay to show that the patient's serum contained disease-causing factors. Cultured human podocytes can be a useful bioassay to monitor disease activity and to screen for podocyte damaging factors. Sera of patients with recurrent FSGS induced downregulation of SMPDL-3b in cultured podocytes making them more susceptible to actin remodeling ${ }^{41}$. We treated cultured human podocytes with the patient's serum and could detect a significant cytoskeleton rearrangement. Actin 
stress fibers in the central part of the cells decreased and the cells displayed a typical actin rim-like structure. These changes are consistent with activation of the cell towards a more motile phenotype that may be more vulnerable to detachment.

In the past, we established the zebrafish as a screening model for proteinuria in gene knockdown models 14-21. Now, we refined our model for a screening of circulating permeability factors. We injected serum of patient with recurrent FSGS in the cardinal vein of the zebrafish and detected a significant loss of plasma proteins 3 days later.

In order to characterize the unknown circulating permeability factor further, we performed Raman spectroscopy in $<50 \mathrm{kDa}$ serum fractions, on kidney biopsies and on podocytes treated with serum of our patient with recurrent FSGS. Raman spectra are directly related to the biochemical composition of tissues 42-44. In the past, Raman was used to detect metabolomic changes in different cancers ${ }^{45,46}$. However, Raman spectroscopy was never used before to study metabolomics in FSGS. Li et al. demonstrated that Raman spectroscopy combined with multivariate analysis can be a potential non-invasive diagnostic tool for nephritis in an anti-GBM mouse model ${ }^{47}$. We previously used Raman to detect cell stress induced by micro particles ${ }^{48}$. As the disease-causing factor was unknown in our patient, we used a global approach based on the ability of Raman to identify spectral markers of the global intrinsic molecular composition. Most prominent differences in Raman peaks between FSGS serum treated and control serum treated cultured human podocytes were found at wave length corresponding to membrane bound phosphatidylcholine, phenylalanine, phospholipids, fatty acids and sphingomyelin.

Small wavelength shifts were present between FSGS serum and control serum treated human podocytes between $700 \mathrm{~cm}^{-1}$ and $800 \mathrm{~cm}^{-1}$. It has been suggested that differences in protein secondary structures might result in a shift of Raman bands ${ }^{49}, 50$. For example, the phenylalanine bands shifted between 997 $\mathrm{cm}^{-1}$ and $1007 \mathrm{~cm}^{-1}$ in different types of collagen. Raman spectra of $<50 \mathrm{kDa}$ serum fraction of the FSGS patient at the time of recurrence corresponded to phospholipids, phosphatidylcholine, L-carnitine and $\mathrm{C}=\mathrm{C}$ lipids confirming a dysbalance in the serum lipoprotein profile.

Raman was able to give a molecular fingerprint on tissue level. Raman signal of the FSGS biopsy again revealed increased membrane bound phosphatidylcholine, phenylalanine, phospholipids, fatty acids and sphingomyelin. Increased Raman peaks in the FSGS biopsy corresponding to phosphatidylcholine, phospholipids and fatty acids were in line with disturbed systemic and renal lipid expression in FSGS 54 . A characteristic Raman band of sphingomyelin was identified at $\sim 1643 \mathrm{~cm}^{-1} 55$. This Raman signal was increased in our FSGS biopsy compared to the preimplantation-biopsy. Raman signal corresponding to Lcarnitine was decreased in the biopsy with FSGS recurrence indicating mitochondrial dysfunction. In line, L-carnitine was reduced in the FSGS serum at the time of disease recurrence in our mass spectrometry analysis. Increased collagen along the Bowman's capsule was reported in FSGS mice and fitting to our Raman measurements with increased signal $1259 \mathrm{~cm}^{-1} 56$. Albumin has major Raman peaks at $830 \mathrm{~cm}^{-1}$, $950 \mathrm{~cm}^{-1}, 1350 \mathrm{~cm}^{-1}$, and $1650 \mathrm{~cm}^{-1} 57$. All these spectra were increased in FSGS relapse biopsy 
compared to 0-biopsy indicating a higher albumin abundance in the damaged kidney due to leakage in the glomerular filtration barrier.

In summary, Raman spectroscopy on serum treated cells, serum fractions and renal tissue was able to identify metabolomic changes in lipoproteins and might reveal novel pathways involved in the pathomechanism of recurrent FSGS.

In addition to Raman spectroscopy, we performed mass spectrometry in the patient's serum at different time points of the disease to characterize the circulating metabolome in FSGS. Metabolite signatures have been demonstrated to possess diagnostic or predictive power for several renal dysfunctions such as acute kidney injury, chronic kidney disease, diabetic nephropathy, kidney cancer, membranous nephropathy, polycystic kidney disease as well as for transplant rejection ${ }^{58}$. Fouque et al. could show that several acyl-carnitines were significantly increased and inversely associated with lower eGFR ${ }^{59}$. Plasma free carnitine concentrations were significantly higher in the acute period of steroid-sensitive nephrotic syndrome compared to the remission period and plasma free carnitine positively correlated with low-density lipoprotein cholesterol, total cholesterol and triglyceride ${ }^{60}$.

Phosphatidylcholine, lysophosphatidylcholine, and sphingomyelin were all described to be elevated in diabetic nephropathy and dysregulation of ceramide metabolism was recently reported to be also involved in diabetic kidney disease ${ }^{61,62}$. Metabolomic profiling of patients with a failing kidney allograft revealed a correlation of serum concentrations of tryptophan, glutamine, dimethylarginine isomers and short-chain acyl-carnitines (C4 and C12) with a reduced GFR ${ }^{63}$. There is emerging evidence that disturbed lipid metabolism might play a role in FSGS. Erkan et al. reported increased fatty acids and phosphatidylcholines as well as reduced phosphatidylcholines in urines from patients with FSGS ${ }^{64}$.

In our analysis, we compared mass spectrometry data of the patient's serum at time of FSGS recurrence to serum of a transplanted control and the FSGS serum before and after CytoSorb treatment at the time of FSGS relapse and at the time of remission. Even though a targeted mass spectrometric approach can only cover a predefined set of metabolites the accuracy and reproducibility is higher compared to profiling approaches and was therefore used in this study. Lysophosphatidylcholines were significantly increased in FSGS serum and decreased after CytoSorb treatment. Well in line, lysophosphatidylcholine 16:0 and 18:0 were also found in a podocyte-selective injury mouse model ${ }^{65}$. Furthermore, podocyte injury-driven lysophosphatidylcholine accelerated glomerular macrophage-derived foam cell infiltration via lysophosphatidylcholine-mediated expression of adhesion molecules and chemokines in glomerular resident cells in FSGS ${ }^{65}$. In addition, phosphatidylcholines were accumulated in the FSGS serum. Urine of patients with FSGS was previously described to contain elevated levels of fatty acids (C16:0, C22:4) and lysophosphotidylcholines (C14:0, C18:1) but decreased levels of phosphotidylcholine (C38:4) compared to healthy subjects ${ }^{64}$.

Serum sphingomyelin was reduced in our FSGS patient in our mass spectrometry analysis. In contrast, Raman spectroscopy revealed increased signal corresponding to sphingomyelin on podocyte and tissue 
level in FSGS. Dysregulation and tissue accumulation of different sphingolipids are typical findings in genetic diseases including Tay-Sachs disease, Fabry disease, hereditary inclusion body myopathy 2 , Niemann-Pick disease, and nephrotic syndrome of the Finnish type ${ }^{66-69}$. Similarly, sphingolipid accumulation has also been reported in glomerular diseases of non-genetic origin including diabetic kidney disease, HIV-associated nephropathy, lupus nephritis and idiopathic FSGS ${ }^{69-71}$. Sphingomyelins are synthesized during the transfer of phosphorylcholine from phosphatidylcholine to ceramide in a reaction catalyzed by sphingomyelin synthase. SMPDL3b, an enzyme that modulates sphingomyelinase activity in podocytes has been shown to be reduced in FSGS ${ }^{72,73}$. Thus, our findings are well in line with a the previously described dysregulation of sphingolipids in FSGS..

Moreover, acylcarnitine that also belongs to the sphingolipid family was reduced at the time of FSGS relapse and increased with CytoSorb therapy. Acylcarnitines were previously described to be reduced in urines from FSGS patients ${ }^{64}$. Acylcarnitines play a role in fatty acid oxidation and transport of acyl-CoA across the inner mitochondrial membrane. Lower acylcarnitine levels are therefore a hint for impaired fatty acid oxidation and mitochondrial dysfunction. Taken together we identified several serum metabolomic signatures involved in lipid metabolism disturbances in FSGS that corresponded to Raman signals of FSGS serum, Raman signal of serum treated podocytes and Raman signals in the biopsy after FSGS recurrence.

These innovative methods might shed new light on the pathogenesis of recurrent FSGS and could be used as a novel tool to predict response to treatment. Metabolic profiling was shown to predict outcome of rituximab therapy in rheumatoid arthritis. Phenylalanine, choline, glycine, threonine and glycerol were all increased in non-rituximab responders versus rituximab responder ${ }^{74}$. Interestingly, all these metabolites were also increases in our FSGS patient that did previously not respond to rituximab when the disease occurred in the native kidneys (table 2 and 3 ). It is tempting to speculate that the CytoSorb therapy changed the metabolites and thus changed rituximab responsiveness after disease recurrence.

In summary, we provide novel evidence for additional circulating factors in FSGS causing early recurrence of the disease in the transplanted kidney. This is supported by the following pieces of evidence: First, the patient had normal levels of previously described circulating factors but rapidly responded to CytoSorb treatment. Second, the patient's serum caused podocyte cytoskeleton rearrangements and proteinuria was induced by injection of the patient's serum in zebrafish. Third, Raman spectroscopy was able to give a molecular fingerprint of recurrent FSGS on serum cell and tissue level and revealed metabolomic changes corresponding to serum mass spectrometry from the patient's serum.

Our findings have several limitations. First, our results were only performed with material of a single FSGS patient. However, we were the first analyzing Raman and mass spectroscopy serially over time in the same patient in serum and kidney biopsies. As idiopathic FSGS is a heterogeneous disease most likely caused by different factors in different patients an individualized approach seems reasonable. Second, we did not actually identify "the" causing disease factor. However, we describe morphological and functional changes induced by the serum and found an altered lipid metabolome associated with 
idiopathic FSGS that might reflect a new subtype of FSGS. The innovative treatment management and analysis methods of this study might be used as a model for personalized treatment approaches and further research on recurrent FSGS. We believe that a patient centric approach is necessary to tailor treatment regimens for individual patients due to the heterogeneity of the disease.

\section{Methods}

\section{CytoSorb apheresis}

CytoSorb apheresis was performed in the department of Nephrology at University of Erlangen. The patient received daily/ weekly CytoSorb apheresis over a cimino fistula on the left forearm. Blood flow rate was $200 \mathrm{ml} / \mathrm{min}$. Anticoagulation during the apheresis was done with $1000 \mathrm{IE}$ Heparin bolus followed by 1000 IE Heparin/h. Patients' blood pressure, heart rate and electrolytes were measured during to the procedure. Venous pressure, arterial pressure and transmembrane pressure were controlled at the site of the apheresis machine. Informed consent was obtained from the participant.

\section{Measurement of circulating permeability factors}

CLCF1 levels in patients' serum were measured with human CLCF1 Sandwich ELISA Kit - LS-F7193 (Catalog \# BMS257, LSBio, Seattle, WA, USA) according to the manufacture protocol. The ELISA can detect levels of CLCF1 between $0.156 \mathrm{ng} / \mathrm{ml}$ and $10 \mathrm{ng} / \mathrm{ml}$. According to the company, healthy subjects have serum levels between $0 \mathrm{ng} / \mathrm{ml}$ and $1.5 \mathrm{ng} / \mathrm{ml}$. Cathepsin $\mathrm{L}$ in patient's serum was measured with human cathepsin L ELISA Kit (Catalog \# BMS257, Invitrogen, Thermo Fisher scientific) according to the manufacture protocol. The ELISA can detect cathepsin L levels between $3.1 \mathrm{ng} / \mathrm{ml}$ and $50 \mathrm{ng} / \mathrm{mL}$. According to the company, healthy subjects have serum levels between $0 \mathrm{ng} / \mathrm{ml}$ and $56 \mathrm{ng} / \mathrm{ml}$. IL-6, sUPAR and SCD25 were measured in the laboratory of the University of Heidelberg. The study was approved from ethic committee of Friedrich-Alexander University Erlangen-Nuremberg (182_19B) and the patient gave written consent. All experiments were performed in accordance with relevant named guidelines and regulations.

\section{Treatment of cultured human podocytes with patient serum}

Immortalized cultured human podocytes were proliferated under permissive conditions at $33^{\circ} \mathrm{C}$. When cultivated at $37^{\circ} \mathrm{C}$, the SV40 T-antigen was inactivated for cell differentiation. Podocytes were differentiated for 10 days on cover slides in RPMI 1640 Medium (Roth, Karlsruhe, Germany) with 10\% fetal calf serum, $1 \%$ Penicillin/Streptomycin and $0.1 \%$ Insulin. At day 7 fetal calf serum was replaced to $10 \%$ control serum or patient serum. Cells were fixed at $0 \mathrm{~h}$ and $6 \mathrm{~h}$ using ice-cold methanol at $-20^{\circ} \mathrm{C}$ for 10 min and permeabelized using $0.1 \%$ Triton for 10 min. After blocking with $10 \%$ donkey serum, immunofluorescent labeling of F-actin was done by incubation with Alexa Fluor ${ }^{\circledR} 546$ phalloidin (Invitrogen) at $4{ }^{\circ} \mathrm{C}$ overnight. Nuclei staining was done with Hoechst. Sides were mounted on glass slides and were visualized under fluorescent microscopy. 
Zebrafish were grown and mated at $28.5^{\circ} \mathrm{C}$. Larvae were kept and handled in standard embryo raising medium, as previously described. Two-day-old zebrafish larvae were anesthetized with $1.5 \% \mathrm{MS}-222$, transferred to an agarose injection mold. Zebrafish were injected with human serum derived from the patient and a healthy control into the cardinal venous sinus using a Drummond Nanoject 200 microinjector. Following injection zebrafish were transferred to embryo raising medium to recovery. Larvae were checked daily until 120 hours post fertilization at which point they were euthanized using $1.5 \%$ MS-222 or 1:500 2-phenoxy-ethanol.

\section{Proteinuria detection in zebrafish larvae}

A transgenic zebrafish line that expresses a fluorescent Vitamin D binding protein $\mathrm{Tg}$ (l-fabp:VDBP:eGFP fish) was used to indirectly measure proteinuria. If plasma proteins are retained in the vascular system, the fluorescent signal from the Vitamin $D$ binding protein increases from over time and can easily be seen in the retinal vessels of the zebrafish. The maximum fluorescence intensities of grayscale images of the pupil of zebrafish larvae that were injected with control serum or patient serum at $48 \mathrm{hpf}$ were measured using Image J (Version 1.48 Wayne Rasband National Institutes of Health, USA) and reported in relative units of brightness.

\section{Metabolome quantification}

Concentrations of 163 metabolites including 13 amino acid/ biogenic amines, sum hexoses, 41 acylcarnitines, 15 lysophosphatidylcholines, 77 phospho- and sphingolipids, and 15 sphingomyelines from serum samples were determined using a targeted metabolomics approach using the Absolute IDQ p150 Kit (Biocrates LIFE Science AG) ${ }^{75}$. Briefly, the liquid chromatography with tandem liquid chromatography with tandem mass spectrometry (LC-MS/MS) analysis carried out by means of multiplereaction monitoring (MRM) acquisition using a Waters Acquity UPLC System coupled with QTRAP 5500 (AB Sciex, Darmstadt, Germany). Raw data were analyzed in Analyst software 1.6.2 (Sciex, Framingham, MA, USA) and processed using MetIDQ software, which is an integrated part of the p150 Kit (Biocrates). Metabolites with $\log _{2}$ ratios above 0.3 or below -0.3 are assumed to be up- or down regulated.

Significance has been computed by two-sited paired Student's $t$-test. Significance has been assumed in cases with p-values below 0.05 .

\section{Generation of serum fractions}

After collection of the whole blood in standard serum tubes, the blood was allowed to clot by leaving it undisturbed at room temperature for $20 \mathrm{~min}$. The clot was separated from the serum by centrifuging at $2000 \mathrm{x} \mathrm{g}$ for $10 \mathrm{~min}$ in a refrigerated centrifuge. Serum was carefully removed by pipetting and stored at $-80^{\circ} \mathrm{C}$ until further fractioning. $100 \mathrm{kDa}, 50 \mathrm{kDa}$ and $10 \mathrm{kDa}$ Amicon Ultra-0.5 mL Centrifugal Filters (Merck) were pre-rinsed with $0.5 \mathrm{ml} 0.1 \mathrm{M} \mathrm{NaOH}$ at $14000 \times \mathrm{g}$ for $30 \mathrm{~min}$ to avoid glycerine interference in the analysis. Next, filters were rinsed with distilled water by spinning $0.5 \mathrm{~mL}$ distilled water for $30 \mathrm{~min}$ at 
$14000 \times \mathrm{g}$. Every 30 min wash was followed by spinning the device in the inverted position at $1000 \times \mathrm{g}$ for $2 \mathrm{~min}$ to remove the residual solution contained in the filter. $0.5 \mathrm{~mL}$ serum was transferred to the $100 \mathrm{kDa}$ filter and centrifuged at $14000 \times \mathrm{g}$ for $30 \mathrm{~min}$. The filtrate of this centrifugation step contained molecules with $<100 \mathrm{kDa}$. The concentrate (remainder of the serum in the filter) was collected by placing the filter device upside down and spinning for $1000 \times \mathrm{g}$ for $2 \mathrm{~min}$. The concentrate contained molecules with $>100$ $\mathrm{kDa}$. The filtrate of the $100 \mathrm{kDa}$ filter was then transferred to the $50 \mathrm{kDa}$ filter and centrifuged at $14000 \times \mathrm{g}$ for $30 \mathrm{~min}$. The filtrate of this centrifugation step contained molecules with $<50 \mathrm{kDa}$. The concentrate of the $50 \mathrm{kDa}$ filter was collected by placing the filter device upside down and spinning for $1000 \times \mathrm{g}$ for 2 min. The concentrate contained molecules with 50-100 kDa. The filtrate of the $50 \mathrm{kDa}$ filter was transferred to the $10 \mathrm{kDa}$ filter and centrifuged at $14000 \times \mathrm{g}$ for $30 \mathrm{~min}$. The filtrate of this centrifugation step contained molecules with $<10 \mathrm{kDa}$. The concentrate of the $10 \mathrm{kDa}$ filter was collected by placing the filter device upside down and spinning for $1000 \times \mathrm{g}$ for $2 \mathrm{~min}$. The concentrate contained molecules with $10-50 \mathrm{kDa}$ and was stored at $-80^{\circ} \mathrm{C}$ until Raman spectroscopy.

\section{Raman spectroscopy and spectral analysis}

Confocal Raman spectra were measured using a LabRam HR Evolution spectrometer (Horiba Scientific) at room temperature under ambient conditions in a backscattering geometry. The biopsies and podocytes were placed on platinum-coated silicon wafers and probed with a $633 \mathrm{~nm}$ HeNe laser focused tightly through a 100x objective (numerical aperture 0.9 ) resulting in a laser beam diameter of $\sim 1 \mu \mathrm{m}$ and a laser power of $\sim 11 \mathrm{~mW}$ on samples. A 300 grooves $/ \mathrm{mm}$ diffraction grating and a charge-coupled device cooled at $\sim 60^{\circ} \mathrm{C}$ were used to detect the Raman scattered light at $1.4 \mathrm{~cm}^{-1}$ resolution. Up to 3600 spectra (substrate excluded) were obtained by mapping for each type of sample: biopsies at time of transplantation and podocytopathy relapse as well as podocytes treated with control and FSGS serum. The serum samples protected by a cover glass were measured with a $532 \mathrm{~nm}$ laser through a $50 \mathrm{x}$ (numerical aperture 0.5 ) and a laser power of $\sim 6 \mathrm{~mW}$. Spectral analysis including polyline baseline fitting, threshold removal of substrate spectra, calculation of mean spectra, and anomaly detection in the range of $350 \mathrm{~cm}^{-1}$ to $1800 \mathrm{~cm}^{-1}$ were performed using LabSpec 6 (Horiba Scientific) and in-house Python scripts.

Formalin-fixed paraffin embedded (FFPE) sections were transferred on silicium coated followed by dewaxing with xylene over a period of 24 hours and replacing the xylene after 20 and 40 minutes. The xylene was then rinsed in isopropanol for $2 \times 2$ minutes and then air dried at room temperature. A Raman spectroscopy map was generated from two measurements belonging to the biopsy at the time of kidney transplantation (0-biopsy) and two measurements belonging to the biopsy at the time of FSGS recurrence in the transplant. In total, 3600 spectra consisting of 1025 different wavelengths were available for images. As all Raman spectra from the biopsies derived from biological material with the same molecular components the pathogenicity was expressed to exclusively cause different ratios in the corresponding Raman so that only small differences in the amplitude of the spectra can be observed. In order to evaluate the difference in Raman spectroscopy quantitatively and with spatial resolution, we used a machine learning approach. An anomaly detection using a One-Class SVM (O-SVM) which has been 
successfully employed in a variety of medical and industrial applications 76 was pursued. O-SVM allows learning a decision boundary or a hypersphere in the high dimensional feature space solely by using the spectra of the physiological samples. Parts of the Raman map, that only contained substrate or noise signal where removed, to not deteriorate the O-SVM and to focus only on the parts of the Raman map that contains signal information. Consequently, the hypersphere approximately matched the distribution of the spectra of the physiological samples. This separation hypothesis was tested with the spectra of the pathological samples (FSGS recurrence). For these Raman spectra, a confidence score could be determined using the O-SVM, which describes whether the spectrum can be considered as anomaly, whereby the degree of the anomaly increases with increasing distance to the hypersphere. The baseline corrected Raman spectra were used as features, which cover a dimension of 1025 wavelengths. To reduce the dimension of the feature vector, principal component analysis (PCA) was further employed so that $95 \%$ of the variance contained within the spectra was covered. This resulted in a dimensionality of 75 PCA components per spectrum, which represent the feature vectors that were subsequently used for generating the decision hyperplane of the One-Class SVM. Consequently, $7200 \times 75$ physiological samples were available for training the O-SVM, as well as potentially 7200 pathological samples for the determination of the anomaly. The parameters of the O-SVM $(v, Y)$ that characterize the decision boundary and balance two properties were empirically set to 0.05 and 0.0005 .

\section{Electron microscopy}

For electron microscopy a piece of the human renal biopsy was fixed overnight in $4 \%$ formalin, post-fixed with $1 \% \mathrm{OsO}_{4}(90 \mathrm{~min}$ ) and stained for $1 \mathrm{~h}$ with $1 \%$ UranyLess (Science services $\mathrm{GmbH}$, Munich, Germany). After dehydration tissue blocks were embedded in Araldite Renlam M1 resin (Serva Electrophoresis $\mathrm{GmbH}$, Heidelberg, Germany). 80nm ultrathin sections were cut on an UC7 ultramicrotome (Leica, Wetzlar, Germany) and rinsed in lead citrate buffer for contrasting before analysis using an Leo912 transmission electron microscope (Zeiss, Oberkochen, Germany).

\section{Statistics}

PCA was performed for Raman analysis as described above. T-test was performed for zebrafish experiments to screen for statistical significance.

\section{Declarations}

\section{Acknowledgments}

The research of this manuscript was funded by "Bundesministerium für Bildung und Forschung (BMBF)" under the project name "STOP-FSGS - Speed Translation-Oriented Progress to Treat FSGS".

\section{Author Contributions}


JMD wrote the manuscript, prepared the figures, performed cell culture and zebrafish work, wrote ethical approval for the manuscript and collected patient samples. JMD and MS designed experiments. GS performed Raman spectroscopy, analyzed the Raman spectra, and prepared the Raman figures, CJ did anomaly detection of the Raman data, SK, and UERK performed mass spectrum analysis CD provided FFPE sections from patient biopsies and ultrastructural pictures done by electron microscopy, AKH helped with zebrafish pictures. SC and MS improved the manuscript with their expert knowledge. All authors reviewed the manuscript.

\section{Competing interests}

The authors declare no competing interests.

\section{References}

1. Kitiyakara, C., Eggers, P. \& Kopp, J. B. Twenty-one-year trend in ESRD due to focal segmental glomerulosclerosis in the United States. Am. J. Kidney Dis. 44, 815-825 (2004).

2. Rosenberg, A. Z. \& Kopp, J. B. Focal Segmental Glomerulosclerosis. Clin. J. Am. Soc. Nephrol. 12, 502-517 (2017).

3. Haas, M., Meehan, S. M., Karrison, T. G. \& Spargo, B. H. Changing etiologies of unexplained adult nephrotic syndrome: a comparison of renal biopsy findings from 1976-1979 and 1995-1997. Am. J. Kidney Dis. 30, 621-631 (1997).

4. De Vriese, A. S., Sethi, S., Nath, K. A., Glassock, R. J. \& Fervenza, F. C. Differentiating Primary, Genetic, and Secondary FSGS in Adults: A Clinicopathologic Approach. J. Am. Soc. Nephrol. 29, 759-774 (2018).

5. Sethi, S., Glassock, R. J. \& Fervenza, F. C. Focal segmental glomerulosclerosis: towards a better understanding for the practicing nephrologist. Nephrol. Dial. Transplant. 30, 375-384 (2015).

6. Lovric, S., Ashraf, S., Tan, W. \& Hildebrandt, F. Genetic testing in steroid-resistant nephrotic syndrome: when and how? Nephrol. Dial. Transplant. 31, 1802-1813 (2016).

7. Savin, V. J. et al. Circulating factor associated with increased glomerular permeability to albumin in recurrent focal segmental glomerulosclerosis. N. Engl. J. Med. 334, 878-883 (1996).

8. Wei, C. et al. Circulating urokinase receptor as a cause of focal segmental glomerulosclerosis. Nat. Med. 17, 952-960 (2011).

9. Maas, R. J. H., Wetzels, J. F. M. \& Deegens, J. K. J. Serum-soluble urokinase receptor concentration in primary FSGS. Kidney Int. 81, 1043-1044 (2012).

10. Cara-Fuentes, G. et al. CD80 and suPAR in patients with minimal change disease and focal segmental glomerulosclerosis: diagnostic and pathogenic significance. Pediatr. Nephrol. 29, 13631371 (2014).

11. Kiffel, J., Rahimzada, Y. \& Trachtman, H. Focal segmental glomerulosclerosis and chronic kidney disease in pediatric patients. Adv. Chronic Kidney Dis. 18, 332-338 (2011). 
12. Mekahli, D. et al. Long-term outcome of idiopathic steroid-resistant nephrotic syndrome: a multicenter study. Pediatr. Nephrol. 24, 1525-1532 (2009).

13. Meliambro, K., Schwartzman, M., Cravedi, P. \& Campbell, K. N. The Impact of Histologic Variants on FSGS Outcomes. Int. Sch. Res. Notices 2014, 913690 (2014).

14. Hentschel, D. M. et al. Rapid screening of glomerular slit diaphragm integrity in larval zebrafish. Am. J. Physiol. Renal Physiol. 293, F1746-50 (2007).

15. Schenk, H., Muller-Deile, J., Kinast, M. \& Schiffer, M. Disease modeling in genetic kidney diseases: zebrafish. Cell Tissue Res. 369, 127-141 (2017).

16. Muller-Deile, J. et al. Podocytes regulate the glomerular basement membrane protein nephronectin by means of miR-378a-3p in glomerular diseases. Kidney Int. 92, 836-849 (2017).

17. Muller-Deile, J. et al. Overexpression of TGF-beta Inducible microRNA-143 in Zebrafish Leads to Impairment of the Glomerular Filtration Barrier by Targeting Proteoglycans. Cell. Physiol. Biochem. 40, 819-830 (2016).

18. Muller-Deile, J. et al. Overexpression of preeclampsia induced microRNA-26a-5p leads to proteinuria in zebrafish. Sci. Rep. 8, 3621-018-22070-w (2018).

19. Muller-Deile, J. et al. Mutation of microphthalmia-associated transcription factor (mitf) in zebrafish sensitizes for glomerulopathy. Biol. Open 8, 10.1242/bio.040253 (2019).

20. Muller-Deile, J. et al. Circulating factors cause proteinuria in parabiotic zebrafish. Kidney Int. 96, 342349 (2019).

21. Schenk, H. et al. Characterizing renal involvement in Hermansky-Pudlak Syndrome in a zebrafish model. Sci. Rep. 9, 17718-019-54058-5 (2019).

22. Xie, J., Farage, E., Sugimoto, M. \& Anand-Apte, B. A novel transgenic zebrafish model for blood-brain and blood-retinal barrier development. BMC Dev. Biol. 10, 76-213X-10-76 (2010).

23. Hanke, N., King, B. L., Vaske, B., Haller, H. \& Schiffer, M. A Fluorescence-Based Assay for Proteinuria Screening in Larval Zebrafish (Danio rerio). Zebrafish 12, 372-376 (2015).

24. Schenk, H. et al. Removal of focal segmental glomerulosclerosis (FSGS) factor suPAR using CytoSorb. J. Clin. Apher. 32, 444-452 (2017).

25. Savin, V. J. et al. Circulating factor associated with increased glomerular permeability to albumin in recurrent focal segmental glomerulosclerosis. N. Engl. J. Med. 334, 878-883 (1996).

26. Zimmerman, S. W. Increased urinary protein excretion in the rat produced by serum from a patient with recurrent focal glomerular sclerosis after renal transplantation. Clin. Nephrol. 22, 32-38 (1984).

27. Chang, J. W. et al. Podocyte foot process effacement in postreperfusion allograft biopsies correlates with early recurrence of proteinuria in focal segmental glomerulosclerosis. Transplantation 93,1238 1244 (2012).

28. Deegens, J. K., Andresdottir, M. B., Croockewit, S. \& Wetzels, J. F. Plasma exchange improves graft survival in patients with recurrent focal glomerulosclerosis after renal transplant. Transpl. Int. 17, 151-157 (2004). 
29. Gallon, L., Leventhal, J., Skaro, A., Kanwar, Y. \& Alvarado, A. Resolution of recurrent focal segmental glomerulosclerosis after retransplantation. N. Engl. J. Med. 366, 1648-1649 (2012).

30. Wei, C. et al. Circulating urokinase receptor as a cause of focal segmental glomerulosclerosis. Nat. Med. 17, 952-960 (2011).

31. Maas, R. J., Deegens, J. K. \& Wetzels, J. F. Serum suPAR in patients with FSGS: trash or treasure? Pediatr. Nephrol. 28, 1041-1048 (2013).

32. Thuno, M., Macho, B. \& Eugen-Olsen, J. suPAR: the molecular crystal ball. Dis. Markers 27, 157-172 (2009).

33. Langkilde, A. et al. Increased plasma soluble uPAR level is a risk marker of respiratory cancer in initially cancer-free individuals. Cancer Epidemiol. Biomarkers Prev. 20, 609-618 (2011).

34. Sharma, M. et al. Janus kinase 2 /signal transducer and activator of transcription 3 inhibitors attenuate the effect of cardiotrophin-like cytokine factor 1 and human focal segmental glomerulosclerosis serum on glomerular filtration barrier. Transl. Res. 166, 384-398 (2015).

35. Savin, V. J., McCarthy, E. T., Sharma, R., Charba, D. \& Sharma, M. Galactose binds to focal segmental glomerulosclerosis permeability factor and inhibits its activity. Transl. Res. 151, 288-292 (2008).

36. Savin, V. J. et al. Renal and Hematological Effects of CLCF-1, a B-Cell-Stimulating Cytokine of the IL-6 Family. J. Immunol. Res. 2015, 714964 (2015).

37. Kemper, M. J., Meyer-Jark, T., Lilova, M. \& Muller-Wiefel, D. E. Combined T- and B-cell activation in childhood steroid-sensitive nephrotic syndrome. Clin. Nephrol. 60, 242-247 (2003).

38. Cocchiaro, P. et al. The Multifaceted Role of the Lysosomal Protease Cathepsins in Kidney Disease. Front. Cell. Dev. Biol. 5, 114 (2017).

39. Yamamoto-Nonaka, K. et al. Cathepsin D in Podocytes Is Important in the Pathogenesis of Proteinuria and CKD. J. Am. Soc. Nephrol. 27, 2685-2700 (2016).

40. Isobe, S. et al. Focal segmental glomerulosclerosis associated with cutaneous and systemic plasmacytosis. CEN Case Rep. 6, 206-209 (2017).

41. Fornoni, A. et al. Rituximab targets podocytes in recurrent focal segmental glomerulosclerosis. Sci. Transl. Med. 3, 85ra46 (2011).

42. Li, J. et al. Raman spectroscopy as a diagnostic tool for monitoring acute nephritis. J. Biophotonics 9, 260-269 (2016).

43. Haifler, M. et al. Discrimination of malignant and normal kidney tissue with short wave infrared dispersive Raman spectroscopy. J. Biophotonics 11, e201700188 (2018).

44. Robichaux-Viehoever, A. et al. Characterization of Raman spectra measured in vivo for the detection of cervical dysplasia. Appl. Spectrosc. 61, 986-993 (2007).

45. Van Nest, S. J. et al. Raman spectroscopy detects metabolic signatures of radiation response and hypoxic fluctuations in non-small cell lung cancer. BMC Cancer 19, 474-019-5686-1 (2019).

46. Larion, M. et al. Detection of Metabolic Changes Induced via Drug Treatments in Live Cancer Cells and Tissue Using Raman Imaging Microscopy. Biosensors (Basel) 9, 5. doi: 10.3390/bios9010005 
(2018).

47. Li, J. et al. Raman spectroscopy as a diagnostic tool for monitoring acute nephritis. J. Biophotonics 9, 260-269 (2016).

48. Sarau, G., Yarbakht, M. \& Kling, L. Context Microscopy and Fingerprinting Spectroscopy of Micro- and Nanoplastics and Their Effects on Human Kidney Cells Using nanoGPS and ParticleFinder. HORIBA technical reports 54 (2020).

49. Steiner, T. \& Koellner, G. Hydrogen bonds with pi-acceptors in proteins: frequencies and role in stabilizing local 3D structures. J. Mol. Biol. 305, 535-557 (2001).

50. Hsu, C. C. et al. A single-cell Raman-based platform to identify developmental stages of human pluripotent stem cell-derived neurons. Proc. Natl. Acad. Sci. U. S. A. 117, 18412-18423 (2020).

51. Kuppe, C., Leuchtle, K. \& Wagner, A. Novel parietal epithelial cell subpopulations contribute to focal segmental glomerulosclerosis and glomerular tip lesions. Kidney Int. 96(1):80-93 (2019).

52. Fatima, H. et al. Parietal epithelial cell activation marker in early recurrence of FSGS in the transplant. Clin. J. Am. Soc. Nephrol. 7, 1852-1858 (2012).

53. Smeets, B. et al. Parietal epithelial cells participate in the formation of sclerotic lesions in focal segmental glomerulosclerosis. J. Am. Soc. Nephrol. 22, 1262-1274 (2011).

54. Wahl, P., Ducasa, G. M. \& Fornoni, A. Systemic and renal lipids in kidney disease development and progression. Am. J. Physiol. Renal Physiol. 310, F433-45 (2016).

55. Shirota, K. et al. Detection of Sphingomyelin Clusters by Raman Spectroscopy. Biophys. J. 111, 9991007 (2016).

56. Schneider, R. R. et al. Compound effects of aging and experimental FSGS on glomerular epithelial cells. Aging (Albany NY) 9, 524-546 (2017).

57. Parachalil, D. R. et al. Raman spectroscopic screening of high and low molecular weight fractions of human serum. Analyst 144, 4295-4311 (2019).

58. Abbiss, H., Maker, G. L. \& Trengove, R. D. Metabolomics Approaches for the Diagnosis and Understanding of Kidney Diseases. Metabolites 9, 34. doi: 10.3390/metabo9020034 (2019).

59. Fouque, D. et al. Relationship between serum carnitine, acylcarnitines, and renal function in patients with chronic renal disease. J. Ren. Nutr. 16, 125-131 (2006).

60. Gousseinov, A., Kantar, M., Mir, S., Keskinoglu, A. \& Coker, I. Free carnitine levels in children with steroid-sensitive nephrotic syndrome. Pediatr. Int. 44, 74-77 (2002).

61. Sas, K. M. et al. Targeted Lipidomic and Transcriptomic Analysis Identifies Dysregulated Renal Ceramide Metabolism in a Mouse Model of Diabetic Kidney Disease. J. Proteomics Bioinform Suppl 14, 10.4172/jpb.S14-002. Epub 2015 May 18 (2015).

62. Suzuki, Y., Fausto, A., Hruska, K. A. \& Avioli, L. V. Stimulation of phosphatidylcholine biosynthesis in diabetic hypertrophic kidneys. Endocrinology 120, 595-601 (1987).

63. Bassi, R. et al. Metabolomic Profiling in Individuals with a Failing Kidney Allograft. PLoS ONE (2017). 
64. Erkan, E., Zhao, X., Setchell, K. \& Devarajan, P. Distinct urinary lipid profile in children with focal segmental glomerulosclerosis. Pediatr. Nephrol. 31, 581-588 (2016).

65. Hara, S. et al. Podocyte injury-driven lipid peroxidation accelerates the infiltration of glomerular foam cells in focal segmental glomerulosclerosis. Am. J. Pathol. 185, 2118-2131 (2015).

66. Haltia, A. et al. Sphingolipid activator proteins in a human hereditary renal disease with deposition of disialogangliosides. Histochem. J. 28, 681-687 (1996).

67. Sango, K. et al. Mouse models of Tay-Sachs and Sandhoff diseases differ in neurologic phenotype and ganglioside metabolism. Nat. Genet. 11, 170-176 (1995).

68. Young, E. et al. Is globotriaosylceramide a useful biomarker in Fabry disease? Acta Paediatr. Suppl. 94, 51-4; discussion 37-8 (2005).

69. Merscher, S. \& Fornoni, A. Podocyte pathology and nephropathy - sphingolipids in glomerular diseases. Front. Endocrinol. (Lausanne) 5, 127 (2014).

70. Haus, J. M. et al. Plasma ceramides are elevated in obese subjects with type 2 diabetes and correlate with the severity of insulin resistance. Diabetes 58, 337-343 (2009).

71. Haus, J. M. et al. Plasma ceramides are elevated in obese subjects with type 2 diabetes and correlate with the severity of insulin resistance. Diabetes 58, 337-343 (2009).

72. Fornoni, A. et al. Rituximab targets podocytes in recurrent focal segmental glomerulosclerosis. Sci. Transl. Med. 3, 85ra46 (2011).

73. Fornoni, A., Merscher, S. \& Kopp, J. B. Lipid biology of the podocyte-new perspectives offer new opportunities. Nat. Rev. Nephrol. 10, 379-388 (2014).

74. Sweeney, S. R. et al. Metabolomic profiling predicts outcome of rituximab therapy in rheumatoid arthritis. RMD Open 2, e000289-2016-000289. eCollection 2016 (2016).

75. Cook, G. W. et al. Structural variation and its potential impact on genome instability: Novel discoveries in the EGFR landscape by long-read sequencing. PLoS One 15, e0226340 (2020).

76. Movasaghi, Z., Rehman, S. \& Rehman, I. U. Raman Spectroscopy of Biological Tissues. Journal Applied Spectroscopy Reviews (2007).

77. Krafft, C., Neudert, L., Simat, T. \& Salzer, R. Near infrared Raman spectra of human brain lipids. Spectrochim. Acta A Mol. Biomol. Spectrosc. 61, 1529-1535 (2005).

78. Mahadevan-Jansen, A. et al. Optical techniques for diagnosis of cervical precancers: a comparison of Raman and fluorescence spectroscopies. Advances in Fluorescence Sensing Technology II (1995).

79. Lieber, C. A., Majumder, S. K., Billheimer, D., Ellis, D. L. \& Mahadevan-Jansen, A. Raman microspectroscopy for skin cancer detection in vitro. J. Biomed. Opt. 13, 024013 (2008).

80. Spiker, R. C.,Jr \& Levin, I. W. Raman spectra and vibrational assignments for dipalmitoyl phosphatidylcholine and structurally related molecules. Biochim. Biophys. Acta 388, 361-373 (1975).

81. Kochan, K. et al. IR and Raman imaging of murine brains from control and ApoE/LDLR(-/-) mice with advanced atherosclerosis. Analyst 141, 5329-5338 (2016). 
82. Mahadevan-Jansen, A. \& Richards-Kortum, R. R. Raman spectroscopy for the detection of cancers and precancers. J. Biomed. Opt. 1, 31-70 (1996).

83. Almeida, M. I. et al. Strand-specific miR-28-5p and miR-28-3p have distinct effects in colorectal cancer cells. Gastroenterology 142, 886-896.e9 (2012).

84. Wiercigroch, E. et al. Raman and infrared spectroscopy of carbohydrates: A review. Spectrochim. Acta A Mol. Biomol. Spectrosc. 185, 317-335 (2017).

\section{Tables}

Table 1: Raman spectra wavelength with corresponding published assignments. 


\begin{tabular}{|c|c|c|}
\hline Raman shift & assignment & reference \\
\hline$\sim 417 \mathrm{~cm}^{-1}$ & chol esterol & 76,77 \\
\hline$\sim 720 / 780 \mathrm{~cm}^{-1}$ & DNA/ RNA & 78,79 \\
\hline$\sim 722 / 718 \mathrm{~cm}^{-1}$ & $\begin{array}{l}\text { membrane bound / free } \\
\text { phosphatidylcholine }\end{array}$ & 80 \\
\hline$\sim 743 \mathrm{~cm}^{-1}$ & thymine & $76,78,79$ \\
\hline$\sim 876 \mathrm{~cm}^{-1}$ & phosphatidylcholine & 81 \\
\hline$\sim 1003 / 1030 \mathrm{~cm}^{-1}$ & phenylalanine & $76,78,79$ \\
\hline$\sim 1002 / 987 \mathrm{~cm}^{-1}$ & $\begin{array}{l}\text { membrane bound / free } \\
\text { phosphatidylcholine }\end{array}$ & 80 \\
\hline$\sim 1125 \mathrm{~cm}^{-1}$ & carbohydrates & 69 \\
\hline$\sim 1125 / 1580 \mathrm{~cm}^{-1}$ & cytochrome c & $76,78,79$ \\
\hline$\sim 1128 \mathrm{~cm}^{-1}$ & $\mathrm{C}-\mathrm{N}$ & 78,79 \\
\hline$\sim 1259 \mathrm{~cm}^{-1}$ & collagen & 78 \\
\hline$\sim 1303 \mathrm{~cm}^{-1}$ & amide III, cytosine, adenine & 82 \\
\hline$\sim 143333 \mathrm{~cm}^{-1}$ & $\begin{array}{l}\alpha(\mathrm{CH} 2 / \mathrm{CH} 3) \text { and } \beta(\mathrm{CH} 2 / \mathrm{CH} 3) \text { of fatty } \\
\text { acid }\end{array}$ & $76,78,79,83$ \\
\hline$\sim 1448 \mathrm{~cm}^{-1}$ & $\mathrm{CH} 2$ protein & $76,78,79$ \\
\hline $1643 \mathrm{~cm}^{-1}$ & sphingomyelin & 55 \\
\hline$\sim 1657 \mathrm{~cm}^{-1}$ & CClipids & $76,78,84$ \\
\hline$\sim 1660 \mathrm{~cm}^{-1}$ & amide I & 76,78 \\
\hline
\end{tabular}

Table 2: Differences in serum metabolites of a transplanted patient with FSGS recurrence versus a transplanted control measured by mass spectrometry. Table shows metabolites that were increased $>0.5$ and decreased $>0.5$ in the FSGS patient competed to the control patient. All values are given as log2 (FSGS patient / stable transplanted control). 


\begin{tabular}{|c|c|c|}
\hline analyte & metabolite class & $\begin{array}{l}\text { increased in FSGS } \\
\text { recurrence versus CTRL }\end{array}$ \\
\hline Orn / Arg & cust. met. indicator & 1,23 \\
\hline Orn & aminoacids & 1,07 \\
\hline lysoPC a C20:3 & glycerophospholipids & 1,04 \\
\hline IysoPC a C18:2 & glycerophospholipids & 0,95 \\
\hline lysoPC a C20:4 & glycerophospholipids & 0,87 \\
\hline Thr & aminoacids & 0,80 \\
\hline PC ae C34:3 & glycerophospholipids & 0,73 \\
\hline PC aa C34:4 & glycerophospholipids & 0,67 \\
\hline PC aa C32:2 & glycerophospholipids & 0,65 \\
\hline SM C22:3 & sphingolipids & 0,62 \\
\hline Ser & aminoacids & 0,60 \\
\hline Gly & aminoacids & 0,57 \\
\hline IysoPC a C17:0 & glycerophospholipids & 0,56 \\
\hline Pro & aminoacids & 0,56 \\
\hline $\mathrm{C2} / \mathrm{CO}$ & cust. met. indicator & 0,52 \\
\hline analyte & metabolite class & $\begin{array}{l}\text { decreased in FSGS } \\
\text { recurrence versus CTRL }\end{array}$ \\
\hline CO & acylcarnitines & $-1,76$ \\
\hline C2 & acylcarnitines & $-0,98$ \\
\hline Tyr / Phe & cust. met. indicator & $-0,88$ \\
\hline C3-DC (C4-OH) & acylcarnitines & $-0,85$ \\
\hline SM C26:0 & sphingolipids & $-0,66$ \\
\hline PC ae C36:0 & glycerophospholipids & $-0,61$ \\
\hline C10:2 & acylcarnitines & $-0,54$ \\
\hline
\end{tabular}

Data is given as $\log 2$ fold change (FSGS recurrence versus con

Table 3: Difference in serum metabolites in FSGS remission versus recurrence measured by mass spectrometry. Table shows metabolites that were initially increased $>0.5$ and decreased more than $>0.5$ as well as metabolites that were initially decreased and increased more than $>0.5$. All values are given as log2 (FSGS patient / stable transplanted control). 


\begin{tabular}{|c|c|c|c|c|}
\hline analyte & metabolite class & $\begin{array}{c}\text { mean at } \\
\text { recurrence }\end{array}$ & $\begin{array}{l}\text { mean at } \\
\text { remission }\end{array}$ & $\begin{array}{c}\text { difference } \text { FSGS remission } \\
\text { versus recurrence }\end{array}$ \\
\hline \multicolumn{5}{|c|}{ decrease ( $>$ deta 0.5 ) in metabolites initially increased ( $>0.5$ compared to CTRL) } \\
\hline IysoPC a C20:3 & glycerophospholipids & 1,00 & 0,20 & $-0,80$ \\
\hline IysoPC a C20:4 & glycerophospholipids & 0,84 & 0,07 & $-0,77$ \\
\hline PC aa C34:4 & glycerophospholipids & 0,73 & 0,03 & $-0,70$ \\
\hline Orn / Arg & Cust. Met. Indicator & 1,35 & 0,70 & $-0,65$ \\
\hline \multicolumn{5}{|c|}{ increase ( $>$ deta 0.5 ) in metabolites initially decreased ( $>0.5$ compared to CTRL) } \\
\hline $\mathrm{CO}$ & acylcarnitines & $-1,72$ & $-0,40$ & 1,33 \\
\hline $\mathrm{C} 2$ & acylcarnitines & $-1,38$ & $-0,39$ & 0,99 \\
\hline SM C26:0 & sphingolipids & $-0,50$ & 0,33 & 0,82 \\
\hline Arg & aminoacids & $-0,67$ & $-0,01$ & 0,66 \\
\hline
\end{tabular}

Data is given as $\log 2$ fold change (mean at FSGS remission versus mean at FSGS recurrence).

Table 4: Difference in metabolites after first CytoSorb versus before first CytoSorb measured by mass spectrometry. Tables shows metabolites that were initially increased $>0.5$ and ddecreased more than $>$ 0.5 as well as metabolites that were initially decreased and increased more than $>0.5$. All values are given as Log2 (FSGS patient / stable transplanted control).

\begin{tabular}{|c|c|c|c|c|}
\hline analyte & metabolite class & $\begin{array}{l}\text { before } 1 . \\
\text { CytoSorb }\end{array}$ & $\begin{array}{l}\text { after } 1 . \\
\text { CytoSorb }\end{array}$ & $\begin{array}{c}\text { difference after versus before } \\
1 \text {. CytoSorb }\end{array}$ \\
\hline \multicolumn{5}{|c|}{ decrease (> deta 0.5 ) in metabolites initially increased ( $>0.5$ compared to CTRL) } \\
\hline Thr & aminoacids & 0,80 & $-0,28$ & $-1,08$ \\
\hline Gly & aminoacids & 0,57 & $-0,11$ & $-0,68$ \\
\hline Ser & aminoacids & 0,60 & $-0,07$ & $-0,67$ \\
\hline Orn & aminoacids & 1,07 & 0,43 & $-0,64$ \\
\hline $\mathrm{C2} / \mathrm{CO}$ & Cust. Met. Indicator & 0,52 & 0,00 & $-0,52$ \\
\hline \multicolumn{5}{|c|}{ increase (> deta 0.5 ) in metabolites initially decreased ( $>0.5$ compared to CTRL) } \\
\hline C10:2 & acylcarnitines & $-0,54$ & 0,19 & 0,73 \\
\hline C3-DC (C4-OH) & acylcarnitines & $-0,85$ & $-0,19$ & 0,66 \\
\hline
\end{tabular}

Data is given as log2 fold change (after 1. CytoSorb versus before 1. Cytosorb).

Table 5: Difference in metabolites after versus before CytoSorb in remission measured by mass

spectrometry. Table shows metabolites that were initially increased $>0.5$ and ddecreased more than $>0.5$ as well as metabolites that were initially decreased and increased more than $>0.5$. All values are given as Log2 (FSGS patient / stable transplanted control). 


\begin{tabular}{|c|c|c|c|c|}
\hline analyte & metabolite class & $\begin{array}{l}\text { before CytoSorb } \\
\text { at time of } \\
\text { remission }\end{array}$ & $\begin{array}{c}\text { after } \\
\text { CytoSorb at } \\
\text { time of } \\
\text { remission }\end{array}$ & $\begin{array}{l}\text { difference after versus before } \\
\text { CytoSorb in remission }\end{array}$ \\
\hline \multicolumn{5}{|c|}{ decrease (> deta 0.5 ) in metabolites initially increased ( $>0.5$ compared to CTRL) } \\
\hline IysoPC a C18:2 & glycerophospholipids & 1,26 & 0,28 & $-0,99$ \\
\hline PC aa C38:1 & glycerophospholipids & 1,02 & 0,06 & $-0,96$ \\
\hline lysoPC a C18:1 & glycerophospholipids & 0,76 & $-0,13$ & $-0,89$ \\
\hline IysoPC a C16:0 & glycerophospholipids & 0,51 & $-0,23$ & $-0,74$ \\
\hline IysoPC a C20:3 & glycerophospholipids & 0,54 & $-0,14$ & $-0,68$ \\
\hline IysoPC a C17:0 & glycerophospholipids & 0,62 & $-0,01$ & $-0,63$ \\
\hline lysoPC a C18:0 & glycerophospholipids & 0,55 & $-0,04$ & $-0,60$ \\
\hline \multicolumn{5}{|c|}{ increase (> deta 0.5 ) in metabolites initially decreased ( $>0.5$ compared to CTRL) } \\
\hline lysoPC a C26:0 & glycerophospholipids & $-1,36$ & $-0,30$ & 1,06 \\
\hline lysoPC a C26:1 & glycerophospholipids & $-0,96$ & $-0,09$ & 0,87 \\
\hline PC aa C24:0 & glycerophospholipids & $-1,10$ & $-0,41$ & 0,70 \\
\hline
\end{tabular}

Data is given as $\log 2$ fold change (after CytoSorb versus before Cytosorb in remission).

\section{Figures}
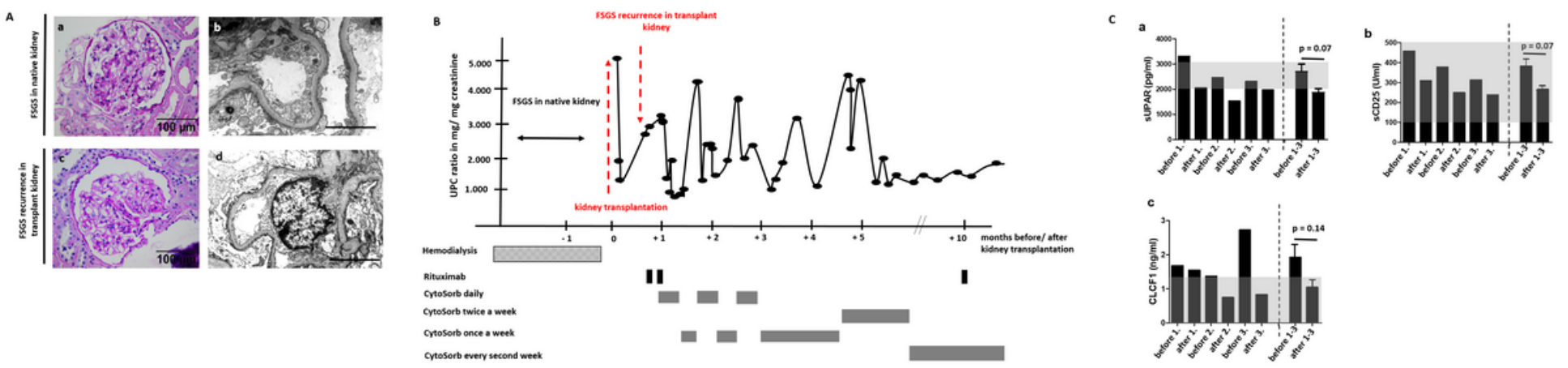

Figure 1

CytoSorb apheresis to treat therapy resistant and early recurrent FSGS. A: a) PAS staining of native kidney biopsy of the patient at the time of initial diagnosis of FSGS. Scale bar $=100 \mu \mathrm{m}$. b) Transmission electron microscopy picture of native kidney biopsy of the patient at the time of initial diagnosis of FSGS. Scale bar $=1 \mu \mathrm{m}$. c) PAS staining of transplant kidney biopsy of the patient at the time of the diagnosis of recurrence of podocytopathy. Scale bar $=100 \mu \mathrm{m}$. d) Transmission electron microscopy picture of transplant kidney biopsy of the patient at the time of the diagnosis of recurrence of podocytopathy. Scale bar $=1 \mu \mathrm{m}$. B: Illustration of clinical course of the patient. Proteinuria measured as urine-proteincreatinine-ratio (UPC-ratio) is given in black dots and lines. Time points of kidney transplantation, transplant kidney biopsy, Rituximab treatment and CytoSorb apheresis schedule are illustrated. C: Measurements for sUPAR (a), sCD25 (b) and CLCF1 (c) in serum samples of the patient before and after first, second and third CytoSorb apheresis. The last two columns depict the mean of measurements for 
the parameters before and after apheresis 1-3. Normal reference levels are shown in gray. Differences before and after CytoSorb apheresis were not significant for sUPAR, CLCF1 and SCD25.

A
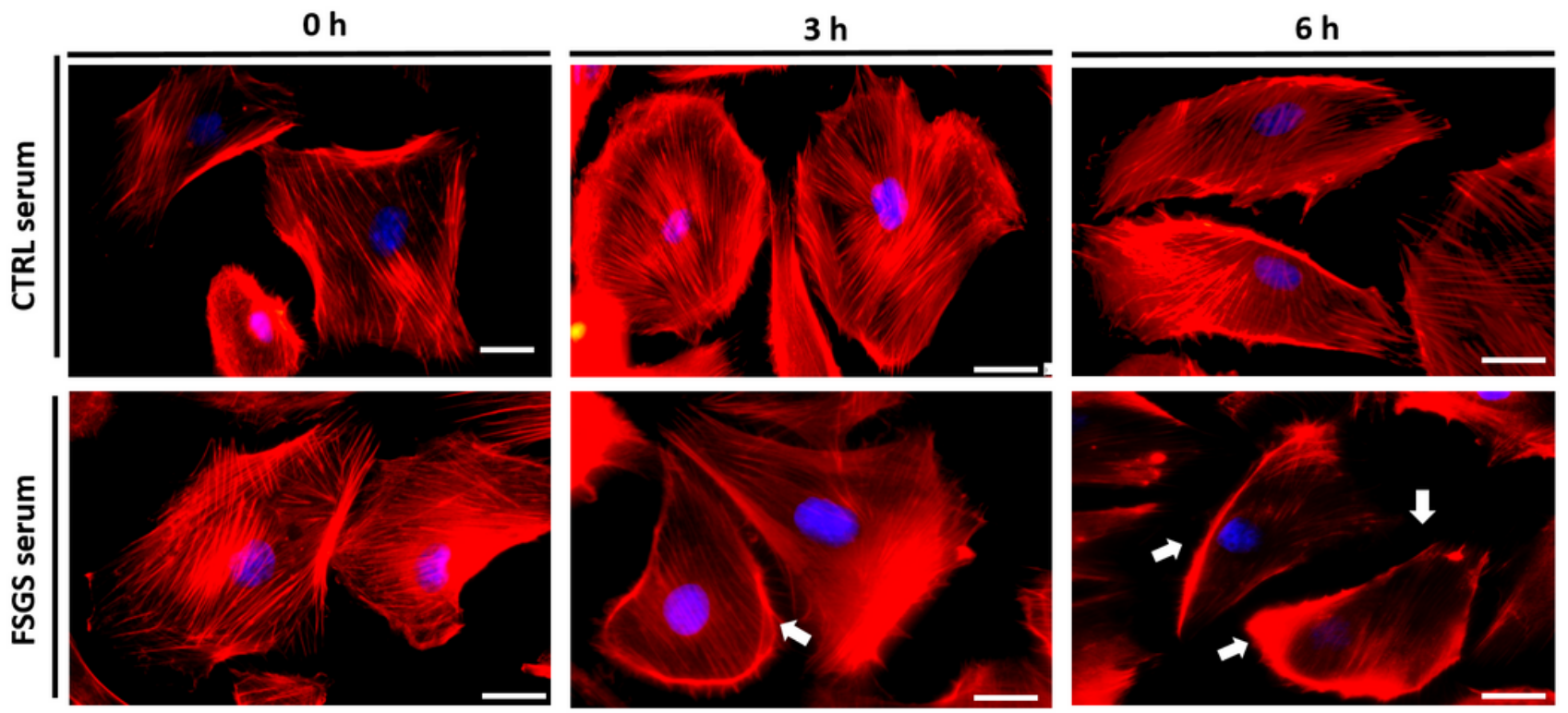

B

$\mathrm{Tg}($ l-fabp:DBP:eGFP) zebrafish
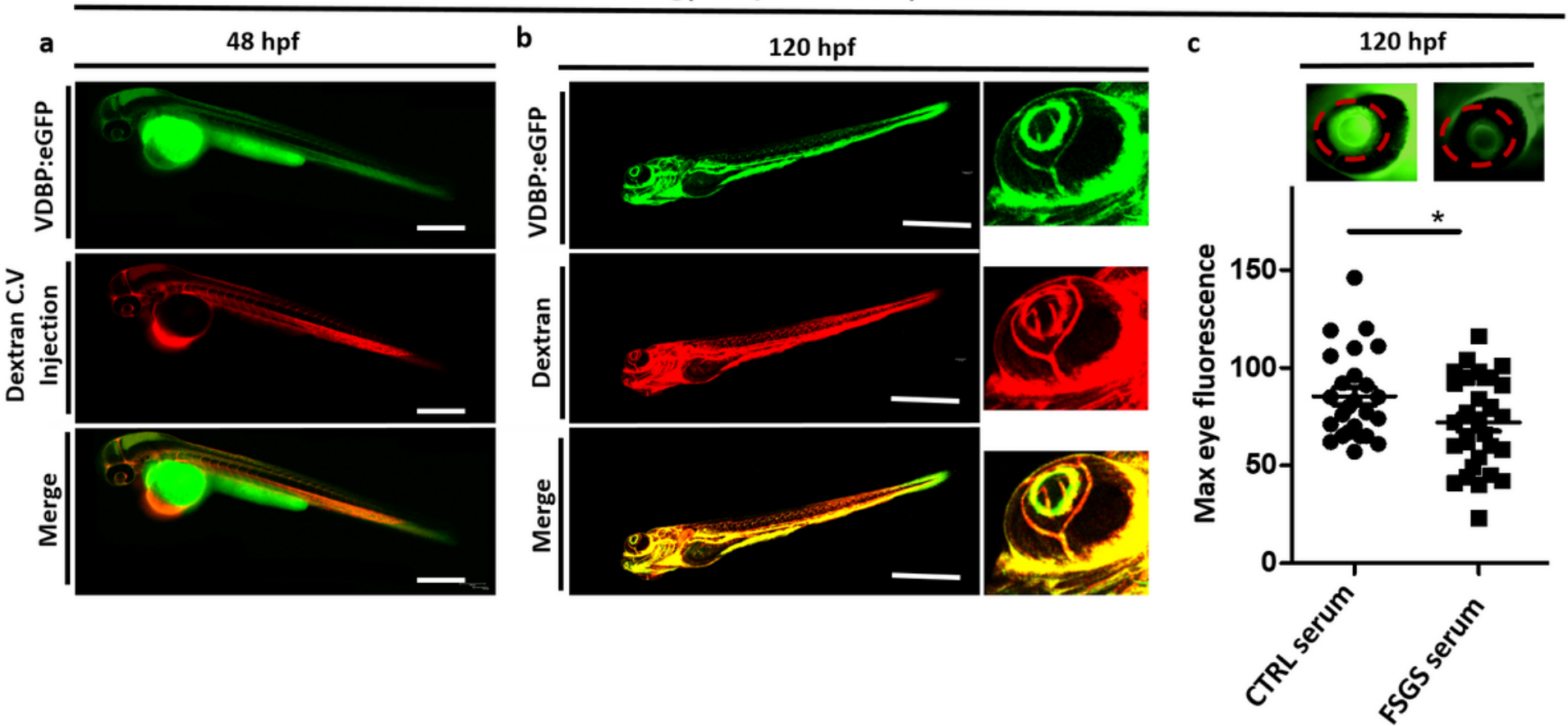

Figure 2

Cell culture and zebrafish assay to detect morphological and functional effects of unknown circulating permeability factors in FSGS A: Representative images of cultured differentiated human podocytes treated with $10 \%$ serum from a healthy control (CTRL serum) and the patient with recurrent FSGS in the kidney transplant (incubation for $0 \mathrm{~h}, 3 \mathrm{~h}$ and $6 \mathrm{~h}$. Cells were stained with phalloidin for cytoskeleton labeling. White arrows indicate cytoskeleton rearrangement. Size bar $=25 \mu \mathrm{m}$. B: Zebrafish assay for the 
detection of circulating permeability factors. a: Representative image of a transgenic $\operatorname{Tg}(\mathrm{I}-$ fabp:VDBP:eGFP) zebrafish larvae (VDBP:eGFP) injected with serum: dextran texas red into the zebrafish circulation (Dextran c.v. injection) at $48 \mathrm{hpf}$. Proper injection leads to red fluorescence of the zebrafish vascularization. Expression of the green fluorescent vitamin $D$ binding protein just started. Size bar $=500$ $\mu \mathrm{m}$. b: At $120 \mathrm{hpf}$ injected Tg(l-fabp:VDBP:eGFP) zebrafish express green fluorescent vitamin $\mathrm{D}$ binding protein (VDBP:eGFP) in the circulation. Red fluorescent serum: dextran mixture is still detectable in the circulation (dextran) and merges with the green fluorescent vitamin D binding protein (merge). The zebrafish eye is enlarged to show the retinal plexus. c: $\mathrm{Tg}(\mathrm{l}-\mathrm{fabp}$ VDDBP:eGFP) transgenic zebrafish can be used to indirectly monitor proteinuria. Loss of green fluorescent protein leads to reduced GFP signal in the retinal vessels of the zebrafish where it can easily be quantified. Quantification of loss of fluorescent vitamin $D$ binding proteins was done by measuring maximum GFP fluorescence in the retinal vessel plexus of $\mathrm{Tg}$ (l-fabp:DBP:eGFP) zebrafish larvae at $120 \mathrm{hpf}$. Zebrafish larvae were injected with serum: dextran mixture from a healthy control and from a patient with FSGS recurrence in the kidney transplant. $*=p<0.05$. hpf: hours post fertilization. $n=107$. Size bar $=500 \mu \mathrm{m}$.

A

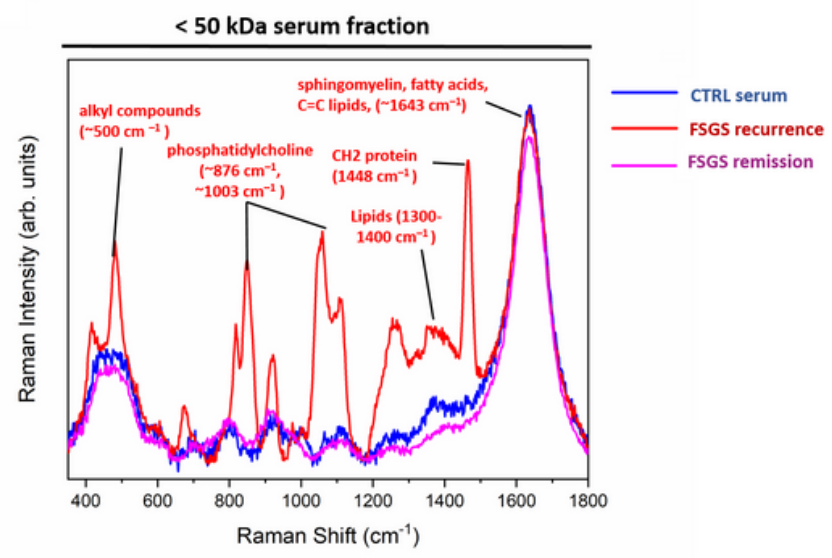

B

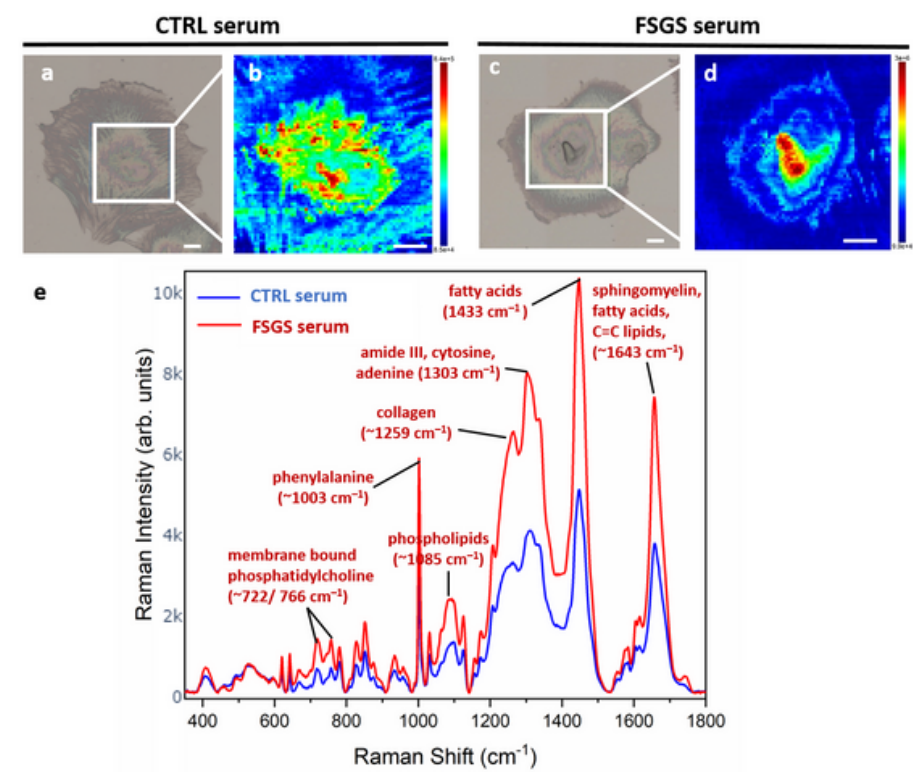

\section{Figure 3}

Raman spectroscopy reveals molecular fingerprint of FSGS serum and changes in podocyte metabolome induced by FSGS serum A: Mean Raman spectra of $<50 \mathrm{kDa}$ serum fractions of FSGS at the time of disease recurrence (red), at the time of remission (purple) as well as of $<50 \mathrm{kDa}$ serum fractions of a control person (blue). Different Raman signal corresponding mostly to lipoproteins were detected at the time of FSGS recurrence. Raman signals at the time of FSGS remission however resembled Raman signals of the CTRL serum fraction. Assignments of the Raman peaks according to the literature are given. B: Representative bright field illumination $(a, c)$ and heat map of Raman signal intensity $(b, d)$ of cultured human podocytes treated with CTRL serum (a, b) and FSGS serum (c, d). Size bar $10 \mu \mathrm{m} . \mathrm{e})$ Mean Raman spectra of three podocytes treated with FSGS serum (red line) and three podocytes treated with CTRL serum blue line) showing increased Raman signal for FSGS treated cells indicating 
A

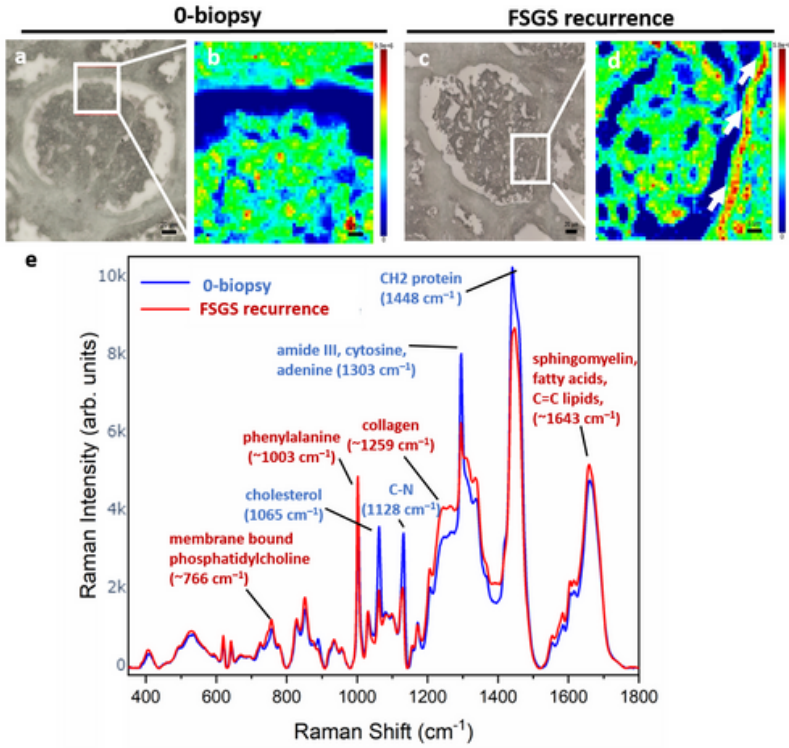

B
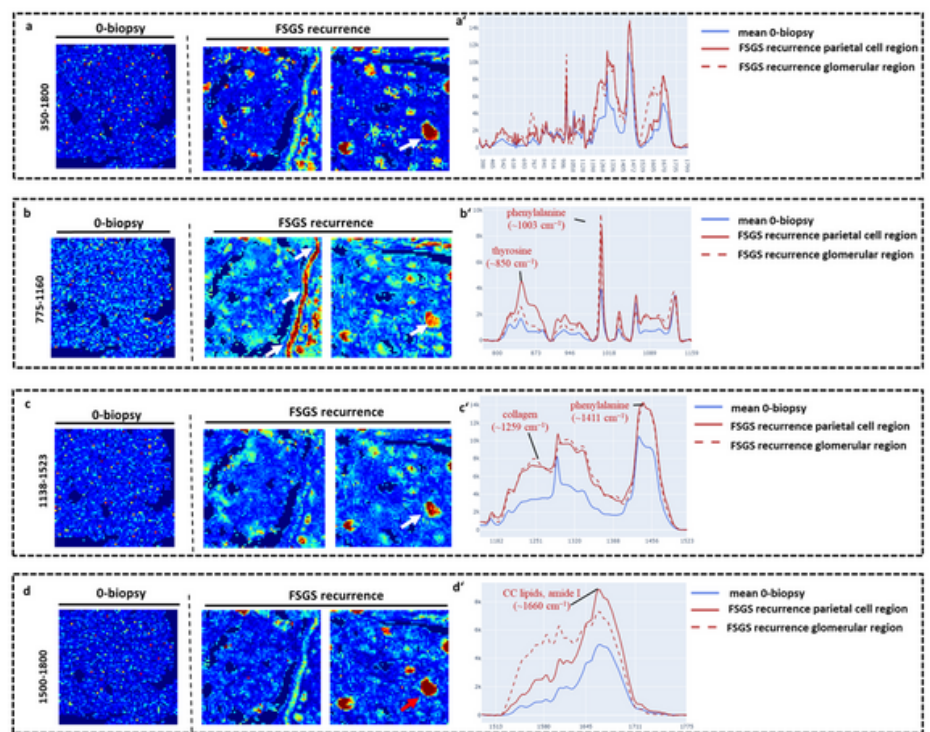

\section{Figure 4}

Raman spectroscopy gives a molecular fingerprint of recurrent FSGS on tissue level A: Representative bright field illumination $(a, c)$ and heat map of Raman signal intensity $(b, d)$ of a glomerulus from the kidney biopsy at transplantation (0-biopsy) $(a, b)$ and at the time of FSGS recurrence (FSGS recurrence) (c, d) showing increased Raman signal at the region of parietal epithelial cell in the Bowman's capsule (arrowhead in c). Scale bar $=50 \mu \mathrm{m}$. e) Mean Raman spectra of three glomeruli from the kidney biopsy at transplantation (blue line) and three glomeruli at the time of FSGS recurrence (red line). Assignments of the Raman peaks according to the literature are given. B: Raman spectra of glomeruli from the 0-biopsy and two glomeruli from the biopsy with FSGS recurrence were visualized, whereby the intensity and color range cover the degree of the anomaly. Areas of focal glomerular lesions as well as parietal epithelial cells in the Bowman capsule are highlighted as an anomaly in the FSGS samples. a: Visualization of the occurring anomaly using the entire spectrum. b-d: Consideration of only parts of the spectrogram that have been baseline corrected individually. Especially in the range of $775-1160 \mathrm{~cm}-1$ (b) there were significant differences in FSGS glomeruli comparison to spectra from glomeruli of the 0-biopsy in focal areas as well in parietal cell region of the bowman capsule. Likewise, a similar effect was observed for the wavelength range 1138-1500 cm-1 (c) and 1500-1800 cm-1 (d). a'-d': Raman spectra for the anomalies with confidence $>80 \%$ in FSGS recurrence of the parietal cell region (red line) and the glomerular region (spotted red line) compared to 0-biopsy (blue line) for the entire spectrum ( $a^{\prime}$ ) and divided in wavelength range of 775-1160 cm-1 (b') 1138-1523 cm-1 (c') as well as $1500-1800 \mathrm{~cm}-1$ (d'). 


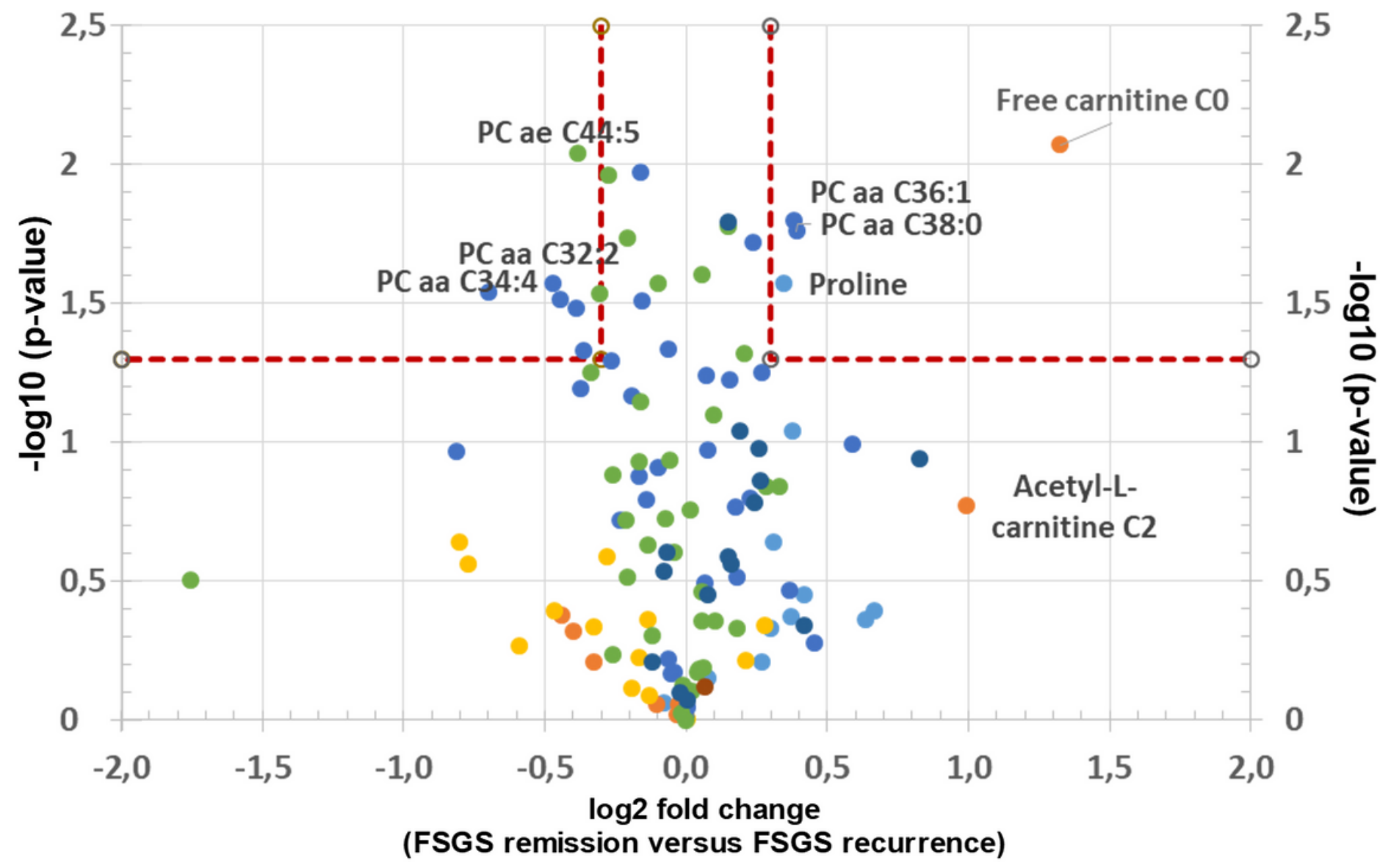

- Amino acids

- lyso PC a glycerophospholipids

- PC ae glycerophospholipids

- sugars
- acylcarnitines

- PC aa glycerophospholipids

- sphingolipids

\section{Figure 5}

Serum metabolome analysis of recurrent FSGS reveals changes carnitine and phosphatidylcholine-levels. Volcano-Plot of the serum metabolome analysis of the patient with FSGS. Log2 fold change of metabolites at FSGS remission versus FSGS recurrence as well as - $\log 10$ (p-values) are given. PCaaC34:4 and L-carnitine were significantly altered at remission versus recurrence. Confidence limits (fold change $>0.3, p$-value $<0.05$ ) are shown with dashed lines. Metabolomics are labeled in different colors according to their metabolomics class.

\section{Supplementary Files}

This is a list of supplementary files associated with this preprint. Click to download.

- supplementarydata.pdf

- supplementarytable1.pdf

- supplementarytable2.pdf 
- supplementarytable3.pdf

- supplementarytable4.pdf 